\title{
Modeling, Enacting, and Integrating Custom Crowdsourcing Processes
}

\author{
STEFANO TRANQUILLINI, FLORIAN DANIEL, PAVEL KUCHERBAEV, \\ and FABIO CASATI, University of Trento, Italy
}

Crowdsourcing (CS) is the outsourcing of a unit of work to a crowd of people via an open call for contributions. Thanks to the availability of online CS platforms, such as Amazon Mechanical Turk or CrowdFlower, the practice has experienced a tremendous growth over the past few years and demonstrated its viability in a variety of fields, such as data collection and analysis or human computation. Yet it is also increasingly struggling with the inherent limitations of these platforms: each platform has its own logic of how to crowdsource work (e.g., marketplace or contest), there is only very little support for structured work (work that requires the coordination of multiple tasks), and it is hard to integrate crowdsourced tasks into stateof-the-art business process management (BPM) or information systems.

We attack these three shortcomings by (1) developing a flexible CS platform (we call it Crowd Computer, or CC) that allows one to program custom CS logics for individual and structured tasks, (2) devising a BPMN-based modeling language that allows one to program CC intuitively, (3) equipping the language with a dedicated visual editor, and (4) implementing CC on top of standard BPM technology that can easily be integrated into existing software and processes. We demonstrate the effectiveness of the approach with a case study on the crowd-based mining of mashup model patterns.

Categories and Subject Descriptors: H.3.5 [Information Systems]: Online Information Services-Webbased services; H.4.1 [Information Systems]: Office Automation-Workflow management; H.1.2 [Information Systems]: User/Machine Systems-Human information processing

General Terms: Design, Languages, Human Factors

Additional Key Words and Phrases: Crowdsourcing, processes, tactics, Crowd Computer, BPMN4Crowd

ACM Reference Format:

Stefano Tranquillini, Florian Daniel, Pavel Kucherbaev, and Fabio Casati. 2015. Modeling, enacting, and integrating custom crowdsourcing processes. ACM Trans. Web 9, 2, Article 7 (May 2015), 43 pages.

DOI: http://dx.doi.org/10.1145/2746353

\section{INTRODUCTION}

Crowdsourcing (CS) is a relatively new approach to execute work that requires human capabilities. Howe [2008], who coined the term, defines crowdsourcing generically as "the act of taking a job traditionally performed by a designated agent (usually an employee) and outsourcing it to an undefined, generally large group of people in the form of an open call." In principle, work could therefore be outsourced in a variety of ways, such as by temporarily recruiting volunteers to help complete a given job or by distributing questionnaires that people fill out voluntarily. However, the environment that has

This work was partially funded by the BPM4People project of the EU FP7 SME Capacities program and by the Evaluation and Enhancement of Social, Economic, and Emotional Wellbeing of Older Adults project under agreement 14.Z50.310029 of Tomsk Polytechnic University, Russia.

Authors' address: S. Tranquillini, F. Daniel, P. Kucherbaev, and F. Casati, University of Trento-DISI, Via Sommarive 9, I-38123 Povo (TN), Italy; emails: \{tranquillini, daniel, kucherbaev, casati\}@disi.unitn.it. Permission to make digital or hard copies of part or all of this work for personal or classroom use is granted without fee provided that copies are not made or distributed for profit or commercial advantage and that copies show this notice on the first page or initial screen of a display along with the full citation. Copyrights for components of this work owned by others than ACM must be honored. Abstracting with credit is permitted. To copy otherwise, to republish, to post on servers, to redistribute to lists, or to use any component of this work in other works requires prior specific permission and/or a fee. Permissions may be requested from Publications Dept., ACM, Inc., 2 Penn Plaza, Suite 701, New York, NY 10121-0701 USA, fax +1 (212) 869-0481, or permissions@acm.org.

(c) 2015 ACM 1559-1131/2015/05-ART7 $\$ 15.00$

DOI: http://dx.doi.org/10.1145/2746353 
contributed the most to the emergence and success of the practice is the Web, and we therefore specifically focus on CS in the context of the Web and on work that is crowdsourced with the help of so-called crowdsourcing platforms. These are online brokers of work (in the form of Web applications/services) who mediate between the crowdsourcer who offers work and the workers who perform work in exchange for a possible reward. The latter form the crowd. Prominent examples of CS platforms are Amazon Mechanical Turk (https://www.mturk.com), CrowdFlower (http://www.crowdflower.com), and 99designs (http://99designs.com). The power of these platforms is their workforce-the crowd-which is potentially large, always available, and can be requested on demand, making the workforce in CS similar to computing power or data storage capability in cloud computing [Baun et al. 2011].

Hoßfeld et al. [2011] statistically analyzed the growth of the Microworkers CS platform from May 2009 to October 2010 and identified a square or a logistic growth model for its crowd. Concretely, according to a recent white paper by Massolution [2013], the enterprise CS market experienced a $75 \%$ growth in 2011, after a 53\% growth in 2010, and was expected to double in 2012. The people engaged as workers in CS platforms span the whole globe, and their number increased by $100 \%$ in 2011 and was expected to grow even more in the years to follow. This is confirmed by the recent first annual survey among enterprise leaders and entrepreneurs by the 2014 Global Crowdsourcing Pulsecheck [Crowdsourcing Week 2014], which reports that about 16\% of respondents think that CS will grow "substantially more than current growth," $38 \%$ say it will grow "more than current growth," and $41 \%$ say it will grow "same as current growth," whereas only 5\% think it will grow "less then current growth." Likewise, the number and type of available CS platforms have enormously grown over the past years, summing up to hundreds of platforms as of today (http://www.crowdsourcing.org/directory) and covering areas, such as Internet services, media and entertainment, technology, manufacturing, financial services, and travel and hospitality. The key benefits identified by crowdsourcers are productivity, flexibility and scalability, cost savings, predictable costs, and better time to market [Massolution 2013].

Typical tasks outsourced on today's CS platforms are so-called microtasks, such as tagging pictures or translating receipts, and creative tasks, such as designing a logo or implementing a piece of software. Each platform approaches these tasks following its very own tactic - that is, organization of work-depending on the nature of the task. For instance, microtasks with small rewards do not require any direct interaction between the crowdsourcer and the worker; creative tasks with higher rewards may instead require a direct negotiation among the two. Therefore, each platform has its own way of describing tasks, publishing tasks, selecting workers, assigning tasks, collecting contributions, assessing quality, rewarding work, and so forth. The tactic thus defines how the platform manages one individual crowd assignment-in other words, an atomic or indivisible piece of work performed by one worker in one transaction.

Composite tasks that require the coordination of multiple individual tasks (assignments of different types of work) typically are not supported. We call these composite tasks crowdsourcing processes, as they require the coordination of multiple individual atomic tasks. Enacting CS processes therefore requires the implementation of ad hoc logic [Kulkarni et al. 2012]. This logic may comprise the specification of a control flow that describes in which order tasks are executed, a dataflow that describes how data is passed among tasks, additional machine tasks that are executed by machines instead of by the crowd (e.g., to aggregate data produced by the crowd), and the like. These requirements make the crowdsourcing of composite tasks highly process driven [Minder and Bernstein 2011; Schall et al. 2012; Kittur et al. 2012; Kucherbaev et al. 2013], hence the name "crowdsourcing process." Platforms such as TurKit [Little et al. 2010b] and Jabberwocky [Ahmad et al. 2011] provide some support for structured CS 


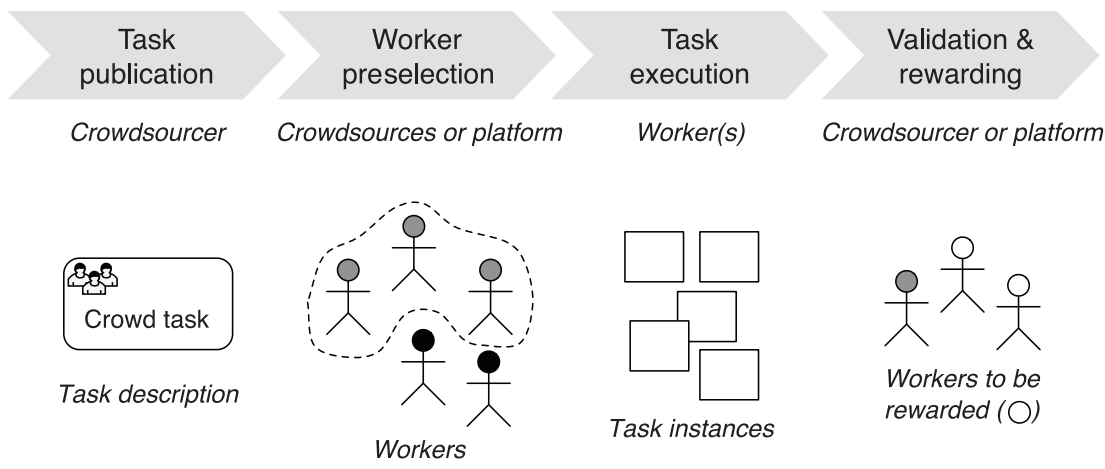

Fig. 1. The high-level steps of CS and the respective actors.

processes to be deployed on top of existing crowdsourcing platforms, typically Mechnical Turk. Yet they suffer from the inherent limitations described earlier and are only hardly integrable with legacy systems (e.g., to enable the inclusion of crowdsourced tasks into common business processes or applications).

These limitations put the healthy growth and further spreading of CS at risk, in that they load the crowdsourcer with unnecessary coordination overhead, prevent scalability, and lower flexibility, as well as threaten cost and time savings if more complex, structured work is to be crowdsourced.

To overcome these limitations, we propose the following contributions:

-A flexible CS platform called Crowd Computer (CC), which allows one to program custom CS logics for individual and structured tasks (an extension of Kucherbaev et al. [2013])

-A business process model and notation (BPMN)-based modeling language equipped with a dedicated visual editor that together allow one to program CC

-A publicly available, open-source implementation of CC on top of standard business process management (BPM) technology that can easily be integrated into existing software and processes and a respective, automated model compiler

-A concrete case study that demonstrates the applicability and effectiveness of the approach in the context of crowd-based mining of mashup model patterns.

The rest of the article is organized as follows. In Section 2, we introduce CS and our problem statement, and in Section 3, we derive requirements and explain how we approach the problem. In Section 4, we present CC, whereas Sections 5 and 6 develop the modeling language for CS tactics and processes. In Section 7, we describe the implementation of our prototype, which we evaluate in Section 8 with the use case. We discuss limitations and related work in Sections 9 and 10, then anticipate some future work in Section 11.

\section{CROWDSOURCING: CONCEPTS AND THE STATE OF THE ART}

CS is a young yet already complex practice, especially with regard to the different ways in which work can be outsourced and harvested. In the following, we conceptualize the necessary background and define the problem that we approach in this article.

\subsection{Crowdsourcing Tasks}

The crowdsourcing of a task using a platform typically involves the steps illustrated in Figure 1 (not all steps are mandatory). The crowdsourcer publishes a description of the task (the work) to be performed, which the crowd can inspect and for which 


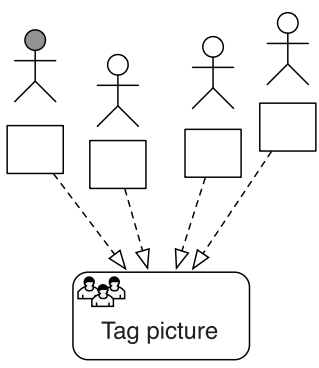

(a) Marketplace task

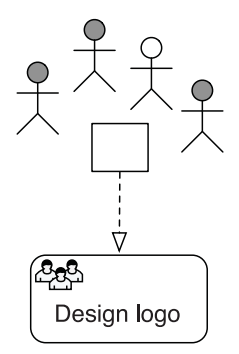

(b) Contest task

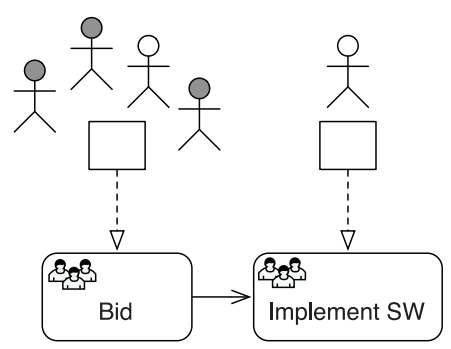

(c) Auction task

Fig. 2. Schemas of the most prominent tactics to crowdsource work.

it possibly can express interest. In this step, the crowdsourcer typically also defines the reward that workers will get for performing the task and how many answers he would like to collect from the crowd. Not everyone in the crowd may, however, be eligible to perform a given task, either because the task requires specific capabilities (e.g., language skills) or because the workers should satisfy given properties (e.g., only female workers). Deciding which workers are allowed to perform a task is commonly called preselection, and it may be done either by the crowdsourcer manually or by the platform automatically (e.g., via questionnaires). Once workers are enabled to perform a task, the platform creates as many task instances as necessary to process all available input data items and/or to collect the expected number of answers. Upon completion of a task instance (or a set thereof), the crowdsourcer may inspect the collected answers and validate the respective correctness or quality. The crowdsourcer typically rewards only work that passes the possible check and is of sufficient quality.

\subsection{Crowdsourcing Tactics}

Depending on the acceptance criteria by both the crowdsourcer and the worker to enter a mutual business relationship (after all, this is what CS is about), different negotiation models may be adopted to crowdsource a piece of work. For simple tasks (e.g., tagging a photo), it is usually not worth it to start a dedicated negotiation process; more complex tasks (e.g., designing a logo or developing a piece of software), instead, may justify a process in which crowdsourcer and worker commonly agree on either the quality of the delivered work or its reward. Since it is the crowdsourcer who starts the CS process and approaches the crowd, we call these negotiation models crowdsourcing tactics. Three major tactics have emerged so far (Figure 2 illustrates the relationship workers-tasks-task instances):

(1) Marketplace: The marketplace tactic [Ipeirotis 2010] targets so-called microtasks of limited complexity, such as tagging a picture or translating a piece of text, for which the crowdsourcer typically (but not mandatorily) requires a large number of answers. Usually, the acceptance criteria by the crowdsourcer for this kind of tasks are simple and clear, such as all answers are accepted (e.g., subjective evaluations or opinions) or only answers that pass a given correctness check are accepted (e.g., a given answer is similar to answers of other workers; a worker answered correct to randomly injected tasks with known answers). Rewards for microtasks commonly range from nothing (workers perform tasks for fun or glory), to few cents or dollars, without any margin for negotiation. If workers find the offer fair, they perform the task; otherwise, they skip it. Prominent examples of CS platforms that implement 


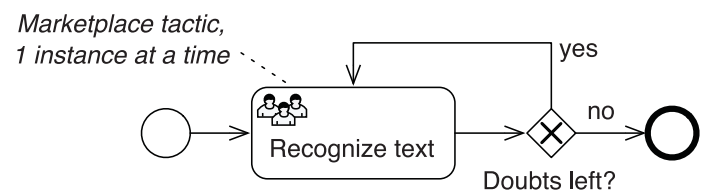

Fig. 3. A simple CS process in BPMN [Object Management Group 2011] inspired by Little et al. [2010b]: the text recognition task is iterated automatically until there are no doubts left about the correct wording.

the marketplace tactic are Amazon Mechanical Turk (https:/www.mturk.com), Microworkers (http://microworkers.com), and CrowdFlower (http://crowdflower.com).

(2) Contest: The contest tactic [Cavallo and Jain 2012] is particularly suitable to creative tasks for which the crowdsourcer knows the budget he is willing to spend but does not have clear criteria to decide which work to accept. Designing a logo and the layout of a Web page are examples of tasks that fall into this category. To enable the crowdsourcer to clarify his criteria, this tactic invites workers to conceive a solution to a task and to participate with it in a contest. Once a given number of contributions or a deadline is reached, the crowdsourcer can inspect all contributions and choose the solution he likes most, thereby electing the winner of the contest (there could be multiple winners). Only the winner is rewarded. Examples of CS platforms that implement the contest tactic are 99designs (http://99designs.com), InnoCentive (http://www.innocentive.com), and IdeaScale (http://ideascale.com).

(3) Auction: The auction tactic [Satzger et al. 2013] targets tasks for which the crowdsourcer has relatively clear acceptance criteria but for which he is not able to estimate a reward. Coding a piece of software is an example of this kind of task. An auction allows the crowdsourcer to publish his requirements and allow workers to express the reward for which they are willing to perform the task. Typically, but not mandatorily (this depends on the adopted auction model), the worker with the lowest offer is assigned the task and is paid accordingly upon delivery of the agreed-on work. An auction can thus be seen as a combination of a contest (to win the auction) and a marketplace task with a predefined worker assignment (to perform the task). An example of an auction-based CS platform is Freelancer (http://www. freelancer.com), which allows programmers to bid for the implementation of software projects.

The latter two tactics aim to produce one result that satisfies the crowdsourcer's need. The marketplace tactic, instead, often aims to produce a large number of results that jointly satisfy the crowdsourcer's need. For instance, the quality of a translation is higher when more workers contribute to it.

In this article, we do not focus on the analysis of which tactic is most effective for which task type (see, e.g., Hirth et al. [2013]). Rather, we aim to enable the flexible design of tactics, which in turn enables answering these and other research questions more efficiently. We specifically do so by focusing on how to aggregate results and coordinate workers, which typically is out of the scope of CS state-of-the-art platforms. We believe that support for different tactics (the preceding three plus custom ones) and for aggregating results and coordinating workers is crucial for the CS of work that is complex, as described next.

\subsection{Crowdsourcing Processes}

Figure 3 shows an example of how to iteratively crowdsource the recognition of a line of text using microtasks until the last worker has no doubts left. As illustrated by the model, translating the text may be more complex than a single task: it may involve multiple instances of the text recognition task and possibly different workers. It 
furthermore requires passing data from one task instance to another, so as to incrementally improve the translation. We call such kind of structuring of multiple crowd tasks and task instances to achieve a common goal a crowdsourcing process.

CS processes are not supported by the CS platforms referenced earlier. The emergence of programming frameworks and higher-level platforms built on top of these CS platforms (most notably, Mechanical Turk) and extending them with basic process management features, however, evince the need for the automation of these kinds of CS processes [Little et al. 2010b; Ahmad et al. 2011; Dean and Ghemawat 2008; Kittur et al. 2011; Kulkarni et al. 2012; Kittur et al. 2012].

The state-of-the-art frameworks/platforms aim to facilitate the CS of individual tasks that require splitting and the coordination of split tasks (e.g., to collect separate feedback for a large number of photos), typically inside an own, proprietary environment. However, there is a set of areas that may benefit from processes that are more advanced than these, such as:

-Product design: Early feedback to new products is crucial to success. Integrating crowd tasks for the collection of feedback, acceptance studies, or testing into production processes may represent a significant competitive advantage.

- Social marketing: Marketing campaigns are increasingly conducted online. The integration of CS into common marketing processes may allow organizations to boost and monitor their social presence.

-Idea management: Increasingly, organizations engage the crowd in the ideation of new products or services. Common social networks do not provide adequate support for this, and idea management systems may be too rigid. Custom CS processes may make the difference.

-e-Democracy: CS may enable the participation of the civil society to politics. How to involve society (e.g., via voting or promoting petitions) is as crucial as election laws. Each party may have its own preferences and goals (i.e., CS processes).

- Human computation: Despite the increasing computing power of machines, there are still tasks that only humans can solve, such as telling whether a portrait photo is beautiful or not. Advanced CS processes enable the flexible integration of both humans and machines, unleashing the computing power of both.

What is missing to bring CS to these areas is (1) support for CS processes that are integrated with common BPM practices [Weske 2007] able to seamlessly bring together the crowd, individual actors, and the machine, and (2) support for crowd tasks with different CS tactics inside a same process, depending on the specific needs of the crowdsourcer. The three tactics described earlier are just the most prominent ones that have emerged so far. They are typically hard coded inside their CS platforms, and each platform has its own tactic with proprietary preselection, quality assessment, and rewarding logics that require a significant amount of manual labor by the crowdsourcer. It is not possible to freely choose and fine-tune how to negotiate a task with the crowd.

The most pragmatic approach to the problem so far is the one by CrowdFlower, which has a set of dedicated CrowdFlower employees working collaboratively with its biggest customers to process data for their most complex jobs/processes. The recent CrowdFlower Labs initiative (http://www.crowdflower.com/blog/introducing-crowdflowerlabs) eventually aims to bring some level of automation to key customers.

The process in Figure 4 illustrates an example of a CS process. It models a logo evaluation procedure where the crowd judges a logo that the crowdsourcer (e.g., a design studio) wants to be assessed. The first two tasks model the upload and evaluation of the logo and respectively are a human task and a crowd task. The third task in the process is a script / machine task to compute the average of the votes that are given by the crowd. The process is closed by a human task where the crowdsourcer inspects the results 


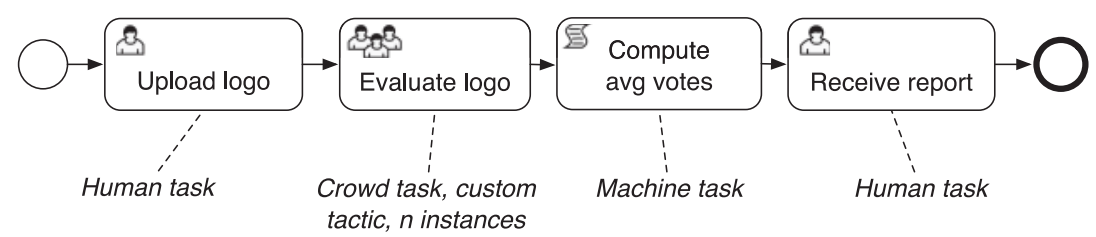

Fig. 4. A CS process involving different actors (humans, machines, and the crowd) and possibly different CS tactics.

of the assessment, the list of the votes, and their average. Human tasks are "typical 'workflow' tasks where a human performer performs the task with the assistance of a software application" [Object Management Group 2011]. Crowd tasks are performed voluntarily by workers of the crowd. The crowd task in the model internally adopts a custom tactic defined by the crowdsourcer that resembles a common marketplace tactic (e.g., without validating results but simply paying all participating workers). The script/machine task is a standard BPMN component that contains a script written by the modeler and later is executed by the BPMN engine.

\subsection{Problem Statement}

The problem that we approach in this article is the design of a model-driven development and execution environment for the implementation of (1) advanced CS processes and (2) custom CS tactics. The work does not focus on any specific application domain (e.g., Hoßfeld et al. [2014a] provide a good overview of CS practices in the domain of multimedia quality evaluation); the work rather aims to provide an enabling technology for the development of flexible, highly tailored CS instruments for all kinds of domains. In addition, we do not further elaborate on how to most effectively describe tasks or how to fine-tune rewards, so as to maximize crowd participation or quality. These are aspects that very strongly depend on the specific task to be crowdsourced, and good studies of the topic already exist [Ipeirotis et al. 2010; Dow et al. 2012; Allahbakhsh et al. 2013].

Next, we summarize the set of core requirements that we identify and explain how we tackle the problem.

\section{MODELING AND ENACTING ADVANCED CROWDSOURCING PROCESSES}

Supporting the modeling and enactment of CS processes is a complex task, especially if the aim is to cater to both CS processes and CS tactics in an integrated fashion and with a similar level of abstraction. We assume that the crowdsourcer interested in this kind of CS support has a working background in business process modeling and management, basic knowledge of CS, and the skills necessary to develop his own Web pages for data collection. For simplicity, we use the term crowdsourcer to refer to both individuals and groups of collaborating individuals with these skills and who want to crowdsource work.

Experience has shown that the CS of any task that is not as trivial as filling out a questionnaire or asking workers to like a Facebook page requires the implementation of custom task logics and designs, typically in the form of Web pages to which workers can be forwarded from within CS platforms. We therefore assume that CS tasks are implemented by the crowdsourcer and that the CS platform (CC) focuses its attention on the coordination of work.

In line with this choice, we further assume that CC does not manage data, as such are directly managed by the CS task pages. CC concentrates on the management of metadata, such as references to the actual data and process runtime data. This allows 
us to keep CC lightweight and the crowdsourcer to not lose control of the data. The alternative would be to also host the actual data on CC. However, this would require the availability of considerable amounts of data storage (expensive) and may imply slow task deployments. More importantly, if CC does not store any potentially sensible data, such as personal or protected data, the crowdsourcer has a higher trust in the platform, and we do not have to worry about the respective legal aspects.

\subsection{Requirements}

Given these assumptions, we derive the following requirements for the implementation of advanced CS processes:

$\boldsymbol{R} 1$ Crowd tasks. The crowdsourcer must be able to properly describe tasks and link them to external task pages.

R2 Metadata exchange. CS task pages must be enabled to exchange metadata with CC, so as to allow CC to coordinate tasks and propagate data.

$\boldsymbol{R} 3$ CS tactics. The tactics used to crowdsource a given task may differ from task to task. The crowdsourcer must therefore be able to design custom tactics, including custom quality assessment and rewarding logics.

$\boldsymbol{R} 4$ Human tasks. These are used when a task has to be executed by a designated human actor (not the crowd), such as the crowdsourcer or an external expert. Supporting human tasks is thus necessary to allow arbitrary human actors to participate in a CS process, such as to validate task outputs.

$\boldsymbol{R} 5$ Machine tasks. Similarly, it is necessary to support machine tasks that enable the integration of computations performed by a machine, such as an operation to compute the average of a series of data extracted from crowd-provided data.

R6 Control flow. Processes are composed of a set of tasks that need to be coordinated. It is necessary to be able to specify the order in which tasks are executed and possible decision points that allow one to split and merge the execution flow.

$\boldsymbol{R} 7$ Dataflow. Tasks may consume data as input and produce data as output. It is thus necessary to enable the crowdsourcer to clearly define which data are produced and consumed by which task and specify suitable data propagation logics.

$\boldsymbol{R} 8$ Data management. CS typically produces large amounts of data. Propagating data among tasks with different data constraints (e.g., show only three photos out of a given set of photos) requires being able to suitably cut, slice, merge, and format data.

Concretely, enabling a crowdsourcer to crowdsource advanced CS processes therefore asks for the design and implementation of the following:

R9 Modeling language. The model-driven design of CS processes with the preceding features requires the conception of a formalism that allows the crowdsourcer to model her own CS processes (BPMN4Crowd).

$\boldsymbol{R} 10$ Modeling editor. To turn the modeling language into an instrument that can also be used in practice, it is necessary to equip the language with a suitable graphical editor that allows the crowdsourcer to model CS processes.

$\boldsymbol{R} 11$ Runtime environment. The execution of CS processes then requires the implementation of a runtime environment that must be able to cater to the preceding features. We call this environment Crowd Computer.

$\boldsymbol{R} 12$ Deployment. Turning a high-level process model into a running process requires the implementation of a dedicated model compiler and support for the automatic deployment of the generated artifacts. 


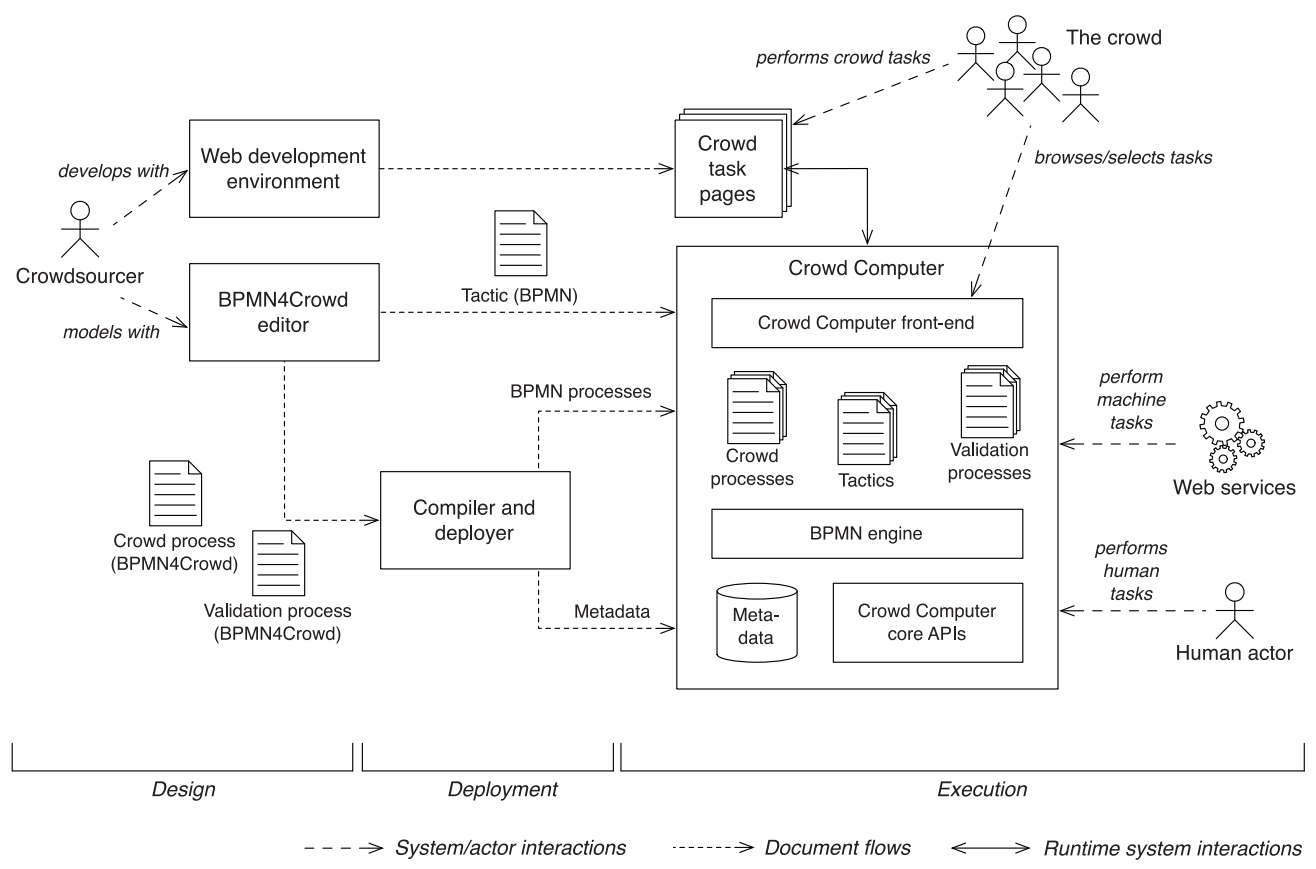

Fig. 5. High-level architecture for the design and enactment of flexible CS processes.

\subsection{Approach}

Figure 5 illustrates our proposed architecture to approach the preceding requirements. It is divided into three parts: design, deployment, and execution.

The design of CS processes starts from the design of CS tasks. These can be implemented by the crowdsourcer as regular Web pages using her preferred Web development environment (R1). For the exchange of metadata between task pages and CC, a dedicated JavaScript task manager API implements the necessary interface (R2). For the modeling of CS processes and tactics, we specifically extend BPMN [Object Management Group 2011]. The rationale of this choice is that BPMN already satisfies some of the requirements outlined earlier: it supports human tasks (R4) and machine tasks (R5), and control flow (R6) and dataflow (R7) constructs, and its native extensibility allows us to implement custom task types for CS tasks (R1) and data management operations (R8), which are not supported by the language. This extended version of BPMN represents BPMN4Crowd (R9), which we accompany with a dedicated BPMN4Crowd editor for the visual design of CS processes and tactics (R10).

BPMN4Crowd is specifically tailored to the needs of CS (the crowd, CS tasks, quality assessment, rewarding, data transformations) and aims to abstract them with intuitive constructs. In Kucherbaev et al. [2013], we proposed a structured approach for the modeling of CS processes, which we refine in this article. Specifically, BPMN4Crowd distinguishes three conceptual modeling layers that foster the separation of concerns:

- CS processes: These are the highest level of abstraction and represent the main processes to be automated. The crowdsourcer models the control flow (R6), dataflow (R7), CS tasks (R1), human tasks (R4), machine tasks (R5), and data transformation tasks (R8) that compose the CS process and configures the tactics of CS tasks (R3) and the exchange of metadata (R2). 
- CS tactics: The second level of abstraction focuses on the tactics (R3). The crowdsourcer decides how to approach the crowd and how to manage crowd tasks. Tactic models express interactions with the APIs of CC (R11).

-Validation and rewarding logics: The third and lowest level of abstraction hosts the models of the different quality assessment and rewarding processes that can be reused when designing tactics (R3).

In this article, we specifically aim to ease the design of these kinds of processes. Tactics and validation/rewarding logics (R3) are particular processes that are not easy to model. We allow the crowdsourcer to develop his own tactics and logics, but we also provide a set of predefined tactics and logics that can easily be reused via suitable configurations of the CS processes. Low-level models serve as libraries to higher levels. We opted for a consistent approach to express logic throughout the whole platform (models) and to allow crowdsourcers to implement their very own validation logics. This acknowledges that we would not be able to predefine all possible validation logics and, at the same time, enhances the reusability of BPMN4Crowd processes.

For the execution of CS processes, we provide CC (R11), which internally uses a standard BPMN engine for process execution plus a set of CC APIs for the management of the CS aspects not supported by the engine, such as metadata management, crowd management, and quality assessment. CC comes with a front-end that can be used by the crowd to discover and perform tasks. The crowdsourcer can start and stop tasks, assign them, approve or reject results, and reward workers, and is able to keep track of work that is assigned, done or pending, and of the performances of each worker. The compiler and deployer automatically translate the models into instructions for CC (R12).

\section{THE CROWD COMPUTER}

CS work requires a CS platform to advertise, assign, perform, and reward work (i.e., CS tasks). With existing platforms, it is not possible to design one's own tactics for tasks, and crowdsourcers have to live with predefined choices (e.g., the type of payment method) and limited customization capabilities, and they are required to use different platforms if they want to crowdsource work using different tactics (e.g., inside a CS process). CC aims to overcome these limitations by (1) singling out as APIs all of the basic features needed to crowdsource work (task, crowd, quality, and reward management); (2) not hardcoding any CS tactic that would in any way limit someone; and (3) enabling the crowdsourcer to assemble her own tactics, coordinating API calls and CS processes on top.

Figure 6 illustrates the internals of CC. The approach to enable the flexible configuration of custom CS tactics is to use a BPMN engine that can be programmed with process models. The latter are stored in a dedicated process repository and may contain tactics, validation, and CS process definitions. The tactics rule the low-level interaction with the internal APIs, whereas the validation and CS processes define possible complex CS logics on top. The APIs maintain internal metadata about tactics and processes in execution. $C C$ front-end acts as interface toward the crowd and the crowdsourcer. It embeds the crowd task pages used to collect results from workers and has a management user interface (UI) for the crowdsourcer.

Running a CS process in CC therefore requires the availability in the process repository of one process model for the CS process, one process model for each tactic used in the CS process, and one process model for each task validation logic used in the tactics. All models are instantiated hierarchically by the BPMN engine, beginning with the CS process model, which is started via CC front-end or the BPMN engine. The task, crowd, quality, and reward manager APIs are called from within the CS process by suitable 


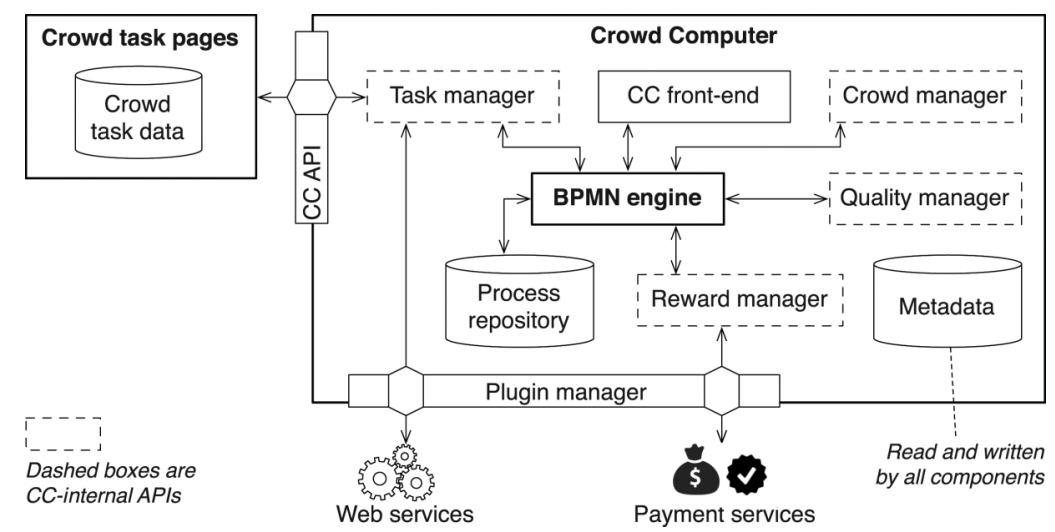

Fig. 6. Functional architecture of CC.

machine tasks. The four APIs all work on an integrated metadata repository, providing for the synchronization of the respective CS operations. Each crowd task inside the CS process causes the BPMN engine to instantiate a new process implementing the respective tactic, which in turn may instantiate a process for the validation of task outcomes. The instantiation of a tactic process causes CC front-end to publish the respective task. This allows workers to pick tasks and perform them through the embedding of the external task pages. Submitting work through a task pages notifies the task manager API and BPMN engine of the completion and progresses the state of the engine and the metadata repository. A CS process terminates when all internally instantiated subprocesses have completed, and now further tasks are to be processed.

We discuss the internal metadata model and APIs next; then we explain the structure and logic of crowd task pages for CC. The following sections explain how to implement tactics and CS processes.

\subsection{Metadata Model}

To support the integration of APIs and the correct execution of tactics and processes, CC implements the metadata model in Figure 7. The model distinguishes the description of tasks, tactics, and processes (instruction register) from their execution (status register). The purpose of the model is to focus on metadata and not on data-that is, CC does not necessarily manage actual data as produced by the workers during task execution. It only keeps track of respective metadata, such as the keys that identify answers by workers inside the crowd task data maintained by the crowdsourcer.

The core element of the instruction register is the TaskDefinition, which contains all information necessary to describe a crowd task, such as name, description, and type (human/machine/crowd). Each task is part of a Process owned by a User. Users have a profile composed of UserProfileParameters (used for preselection) and are connected to task definitions through a UserRole, which tells which role a user plays in which process (e.g., for human tasks). Task definitions comprehend the ParameterTypes of their configuration and runtime parameters (e.g., for the invocation of machine tasks or for control flow decisions), as well as the DataObjectTypes and DataPropertyTypes of the inputs and outputs consumed/produced by the task execution. Data objects store references to the external crowd task data repository and enable data splitting and merging inside CC. Data properties enable the association of descriptive information to data objects and enable, for example, the definition of custom quality controls and the correlation of task instances if multiple instances work on a same object. 


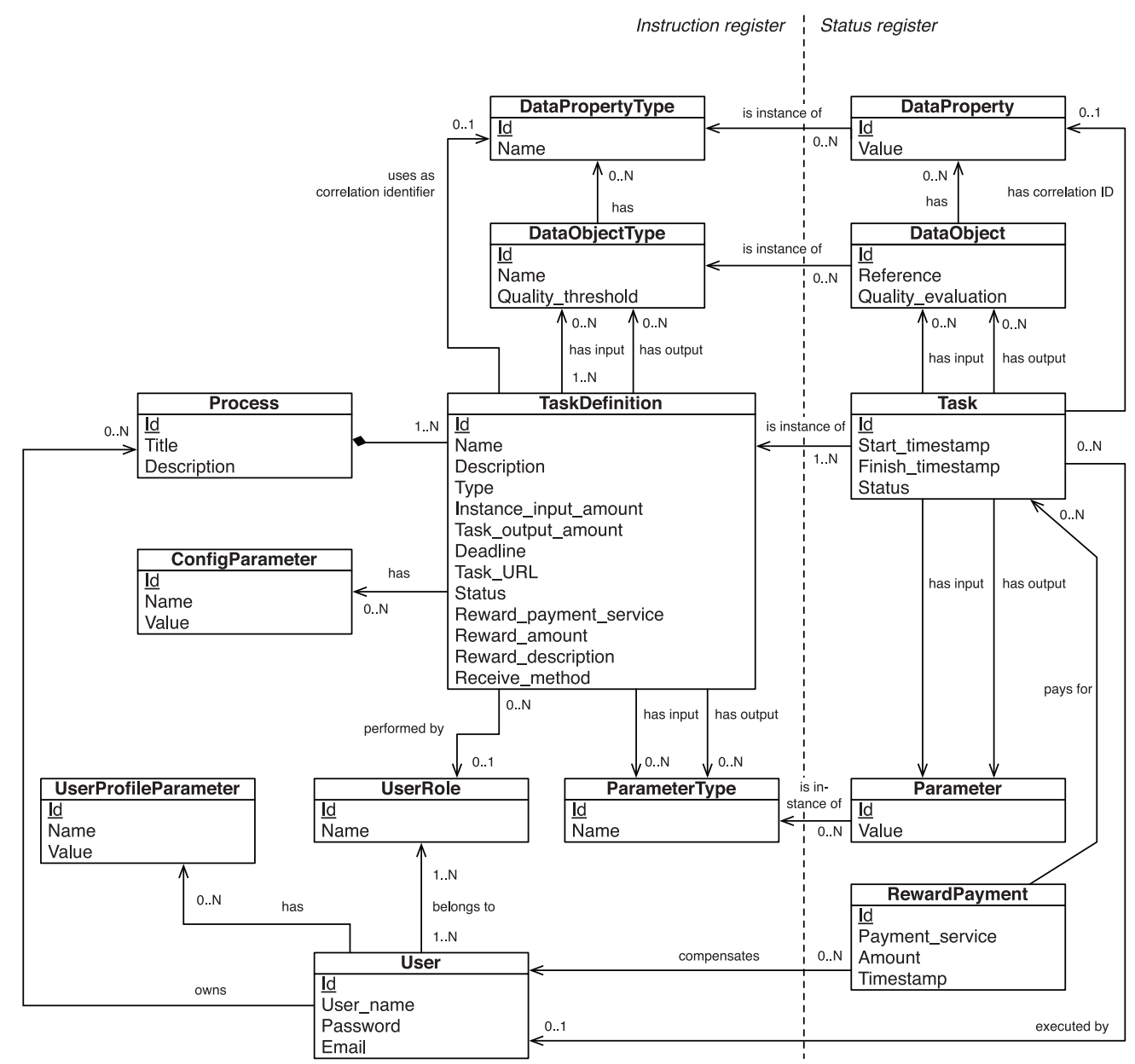

Fig. 7. Metadata model underlying the execution of tasks, tactics, and processes in CC.

The status register contains the runtime data. The Task represents a task instance, it contains information about the task status and is associated to a user (e.g., a worker). In addition, it is associated with the set of Parameters and DataObjects carrying concrete input and output data, as well as the DataProperties describing the produced data objects. The RewardPayment stores information about the reward that is given to workers, which can be used with different payment_services.

\subsection{Programmable APIs}

The metadata modeled in Figure 7 is created and consumed by the processes running in the BPMN engine (tactics, validation, and CS processes), mediated by the internal APIs of CC. In other words, CC can be "programmed" via process models coordinating the invocation of API operations representing the "instruction set" of CC. The goal of $\mathrm{CC}$ is to keep the instruction set focused to the core CS aspects and hence simple, manageable, and efficient. The four APIs are as follows (see Figure 6):

-Task manager: The task manager comprises a set of operations for task life cycle management and data propagation among tasks. Operations are instantiating tasks, 
assigning tasks to workers, canceling tasks in execution, rerunning them, enacting machine tasks, and so forth. Each task can be instantiated multiple times, so as to collect the amount of data that the task is required to produce as output. The API parses the task definitions with their configuration parameters, runtime parameters, and input and output data object specifications, as well as manages the respective runtime values.

- Crowd manager: The crowd manager is in charge of human resource management and worker preselection. It comprises a set of operations for the management of users, such as resolving user roles, preselecting potential workers, tracking which user executed which task, sending direct invitations to people, ensuring separation of duties, and so forth.

-Quality manager: Typically, but not mandatorily, only work that passes a quality assessment is rewarded. The quality manager provides for the necessary management of quality metadata for crowd tasks. It allows one to (1) represent quality as a normalized value in a range from $0(\min )$ to $10(\max ),(2)$ set a minimum threshold level below which work is rejected and not rewarded, and (3) assign quality assessments to task instances at runtime. How to set threshold levels and assess quality (i.e., the semantics of quality) is decided by the crowdsourcer at design time and assessed via custom human/machine or CS tasks at runtime, respectively.

- Reward manager: The reward manager provides for payment management and is in charge of keeping track of which task instances have been rewarded (i.e., paid) and how. Each task may have an associated reward and payment service, and payments may occur for individual task instances, bundles of task instances, and so forth. CC does not impose any reward logic; it can be specified ad hoc or instantiated from reusable model templates. Additionally, CC does not impose any concrete payment platform (e.g., PayPal, VISA); such can be chosen and plugged in by the crowdsourcer. Again, the semantics of the Reward_amount property of tasks is decided by the crowdsourcer.

In Table I, we summarize the most important operations accessible via the APIs that we use throughout this article. Discussing all operations is beyond the scope of this article, so we refer the reader to http://apidocs.crowdcomputer.org for the complete details.

\subsection{Crowd Task Pages}

The front-end of CC is implemented as a regular Web application. For the front-end to be able to visualize a task page, which is also a Web application, it must be published online and embeddable into an iframe inside the front-end's own UI.

In the most simple case, a task page is a static HTML Web form hosted online. For more complex task pages with their own application logic, the application logic should be integrated with that of CC to enable the exchange of data and/or metadata, as described in Figure 8. To support this integration, the task pages must contain a task manager client (a JavaScript library in the current implementation). This client allows the task page to communicate with the task manager API of the embedding CC front-end. Specifically, when a task is instantiated and its UI is embedded into the iframe, the task manager API loads from the metadata store the data identifiers of the input data objects to be processed by the task page (if inputs are to be processed) and sends them to the task manager client. The task page receives the identifiers, loads the corresponding data from its internal data store, and displays them to the worker. Upon the completion of a worker's feedback, the task page stores the results in its data store and sends the respective result identifiers back to the task manager API, which in turn stores them into the metadata store and marks the instance as executed. 
Table I. Excerpt of the Most Important Operations of CC APIs

\begin{tabular}{|c|c|c|c|c|}
\hline API & Operation & Input & Output & Description \\
\hline \multirow[t]{9}{*}{ Task } & create & $\begin{array}{l}\text { Description, task page } \\
\text { URL, number of } \\
\text { instances, deadline, } \\
\text { validation strategy, } \\
\text { reward, reward } \\
\text { strategy, preselection } \\
\text { condition }\end{array}$ & task id & $\begin{array}{l}\text { Creates a task in CC's instruction } \\
\text { register. }\end{array}$ \\
\hline & start & task id & - & $\begin{array}{l}\text { Starts a task and makes it visible to } \\
\text { the crowd. }\end{array}$ \\
\hline & stop & task id & - & $\begin{array}{l}\text { Stops a task and makes it invisible to } \\
\text { the crowd. Pending feedback for } \\
\text { stopped tasks is not accepted. }\end{array}$ \\
\hline & createInstance & task id & $\begin{array}{l}\text { task } \\
\text { instance } \\
\text { id }\end{array}$ & $\begin{array}{l}\text { Creates an instance of a task, starts } \\
\text { it, and makes it available to workers } \\
\text { for execution. }\end{array}$ \\
\hline & stopInstance & task instance id & 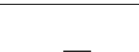 & Stops the specified instance. \\
\hline & assignInstance & $\begin{array}{l}\text { task instance id, user } \\
\text { id }\end{array}$ & - & Assigns a task instance to a user. \\
\hline & storeResult & task instance id, data & - & $\begin{array}{l}\text { Stores the data for the specified task } \\
\text { instance. }\end{array}$ \\
\hline & updateInstance & task instance id, data & - & $\begin{array}{l}\text { Updates the status of the specified } \\
\text { instance. }\end{array}$ \\
\hline & updateInstances & $\begin{array}{l}\text { task instance ids } \\
\text { (array), data }\end{array}$ & - & $\begin{array}{l}\text { Updates the status of all specified } \\
\text { instances. }\end{array}$ \\
\hline Crowd & preselect & user id, task id & true/false & $\begin{array}{l}\text { Runs the preselection logic for the } \\
\text { given user to check if he or she is } \\
\text { allowed to perform the given task. }\end{array}$ \\
\hline Quality & set & task instance id, data & - & $\begin{array}{l}\text { Assigns the quality-level data as } \\
\text { quality assessment to the selected } \\
\text { instance. }\end{array}$ \\
\hline Reward & give & user id, reward & - & Assigns a reward to a worker. \\
\hline
\end{tabular}

Note: Each API implements additional operations that are not shown here. In this table, we concentrate on those operations that are used to model the processes presented later in the article.

\section{MODELING CROWDSOURCING TACTICS}

In the introduction to this article, we stated that a crowd task is atomic or indivisible, referring to the perspective of the worker who executes the crowdsourced work. Yet it is important to note that enacting a crowd task in practice is not an atomic action executed only by a single actor. It rather is a composition of different tasks possibly executed by multiple actors, namely the worker, the crowdsourcer, and the platform. We call this composition the tactic, and it models how to crowdsource a given task.

Throughout this article, we introduce BPMN4Crowd, an extension of BPMN specifically tailored to the needs of CS, and show how to develop custom tactics. First, we provide a summary of the basis of BPMN.

\subsection{Background: BPMN}

BPMN [Object Management Group 2011] is a standard for business process modeling that provides a graphical, flowcharts-based notation to specify business process 


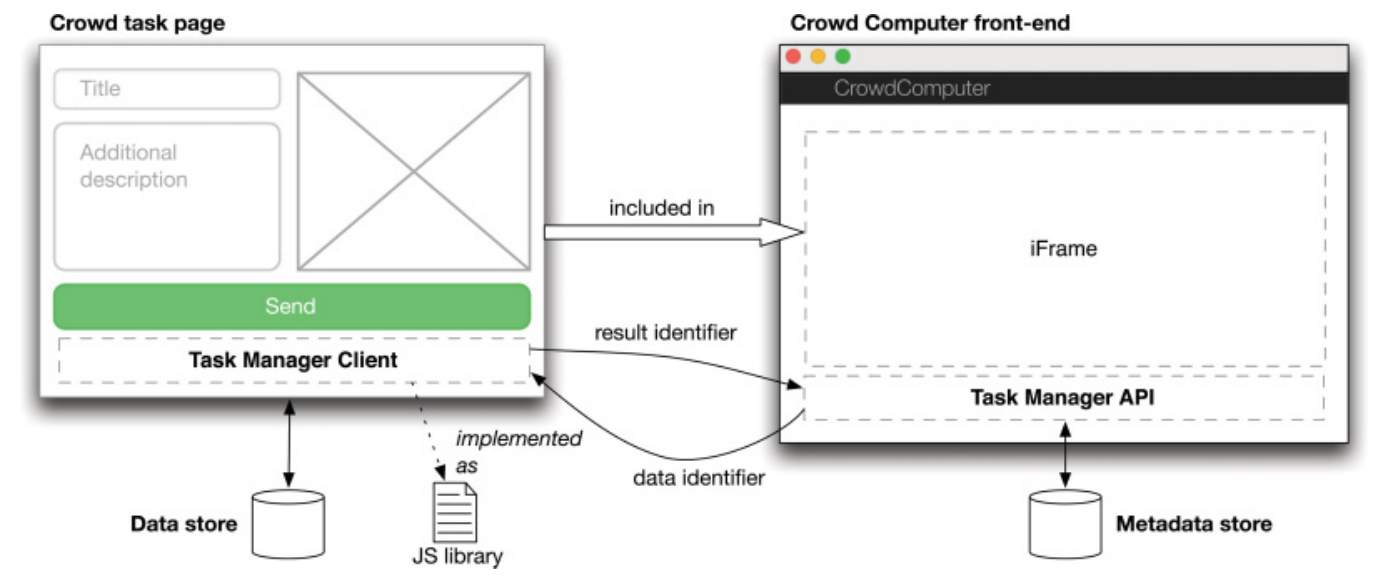

Fig. 8. Implementation logic of task pages to be included in and managed by CC.

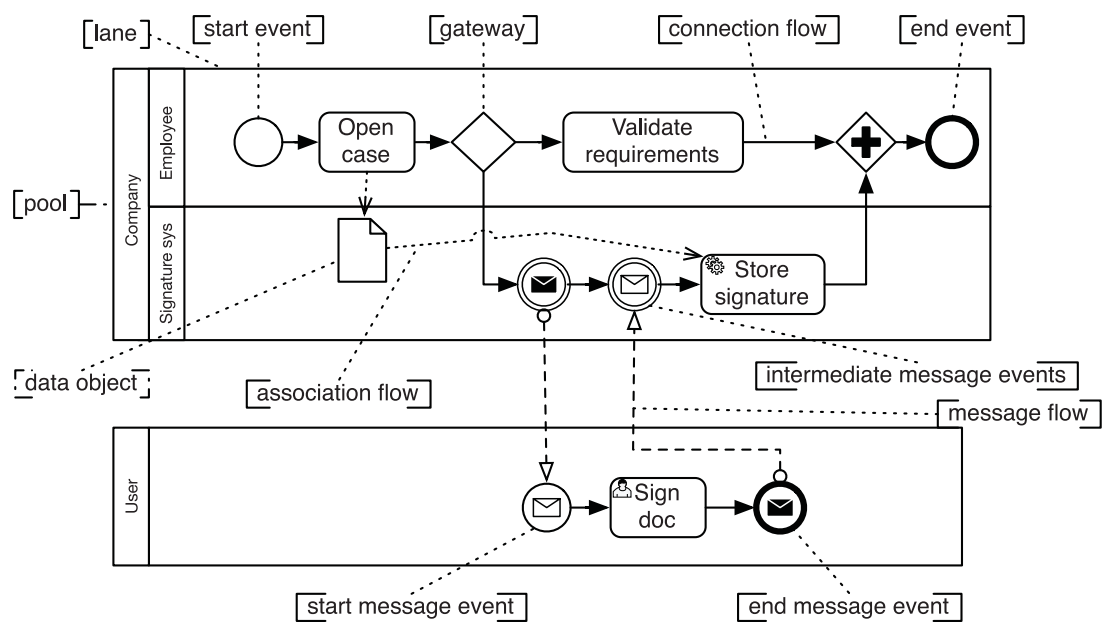

Fig. 9. The core constructs of a BPMN process diagram.

diagrams. Processes are used to model the interactions and business operations that actors have to perform to achieve a given goal. The BPMN notation is composed of four classes of components: flow objects, connecting objects, swim lanes, and artifacts.

Figure 9 illustrates an example of a BPMN process. The model shows a process in which an insurance company opens a new case for a hypothetical user. Inside the pool company, there are two lanes that represent two actors. The first is the employee who opens the case and validates the requirements. The second lane models the signature system, which stores the signature given by the user. The second pool models the user's actions: he waits for a notification telling him to sign a document, signs it, and sends the signature back to the signature system. When the signature is stored and the requirements are validated, the process ends.

This small examples helps us introduce the BPMN constructs that are used in this article, concretely the following classes. Flow objects contain the main elements to create a process: events, activities, and gateways. Events affect the control flow-they have a cause and an impact and are used to start or end a process and to intercept or trigger intermediate events; their shape is a circle. Activities are used to model the work to be performed (i.e., the tasks). There are various types of tasks: among them 


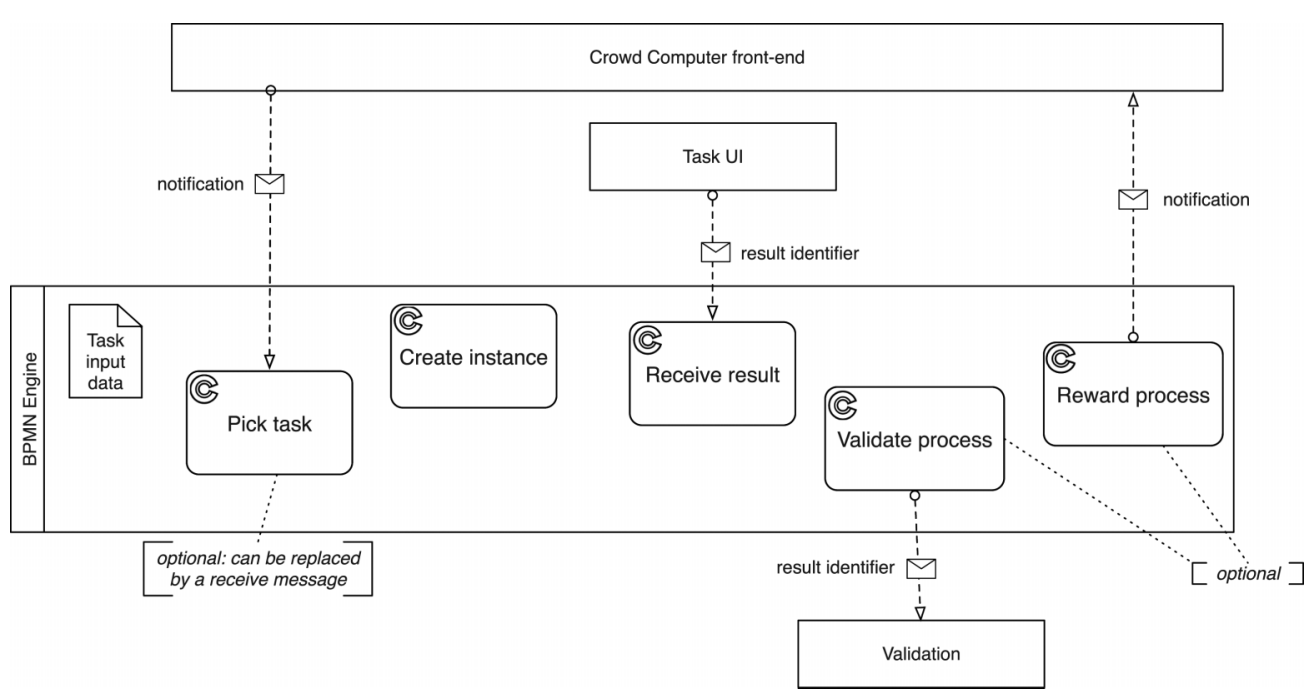

Fig. 10. The basic structure of BPMN4Crowd process diagrams for tactics. Please note that the model is not a correct, executable BPMN model; it is a template that needs to be instantiated into a correct model.

are human tasks, executed by a person, or machine tasks, executed, for example, by a Web service; the shape of tasks is a rounded rectangle with an icon in the top-left corner that identifies the type of task. Gateways represent decision points, such as xor or and conditions; their shape is a diamond. The group of connecting objects contains elements that represent sequences, messages, and dataflows by means of arrows. The sequence (control) flow is modeled with a solid line; the message flow, which models a message exchanged among process participants, is a dashed arrow; the association, used to associate data and artifacts with a task (thus the data flow), is a dotted arrow. Swim lanes (short lanes) separate the activities of different process participants (also called actors). Pools group lanes/participants into organizations (e.g., a company or institution). Artifacts are additional elements of the diagram that are used to add context (e.g., a descriptive comment). The most important element of this category is the data object, which shows how and which data is consumed or produced by the tasks of the process.

\subsection{Basic Structure}

Modeling a CS tactic requires complying with a set of conventions that guarantee CC is provided with all of the information required to correctly publish, assign, collect, assess, and reward work. We summarize these conventions in the basic tactic structure (a template) depicted in Figure 10. Note that we mark CC API invocations with a CC logo in the top-left corner; technically, these tasks correspond to common machine tasks, but for a better readability of the models, we explicitly distinguish those tasks that interact with CC-internal APIs from those that interact with humans, external services, or the crowd.

The process diagram is articulated into four pools, an input data artifact, and a minimum set of tasks that invoke the operations of CC APIs. The pools correspond to the BPMN engine orchestrating the tactic, CC front-end mediating between the tactic and the crowd, the task UIs (crowd task pages) for performing work, and the validation logics. The task input data carries the input data for the task to be crowdsourced. The five tasks serve the following purpose: 


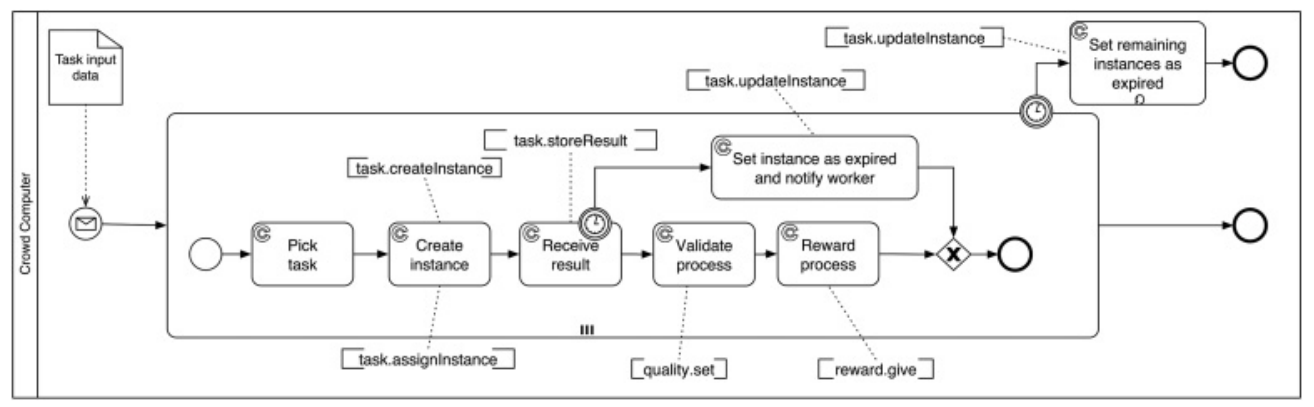

Fig. 11. Simplified process diagram of the marketplace tactic with multiple workers performing tasks in parallel. For a better readability, we omit the input/output data objects, data-flows, and API call details.

- Pick task (no API call need, optional) receives the identifier of the worker that decides to work on a given task listed in CC front-end. The construct is a shorthand for the specification and configuration of a receive message event. Skilled modelers can directly use any of receive message events of the BPMN (e.g., message start event or message receive event).

- Create instance (Task.createInstance and Task.assignInstance, mandatory) uses the task metadata from CC's internal data store to create an instance of the task and assign it either to the worker identified via a prior Pick task or by automatically assigning a worker (e.g., via email).

-Receive result (Task. storeResult, mandatory) represents the actual execution of the task instance. It interacts with the task UI and manages the data identifiers of the task inputs and outputs (metadata) as explained earlier.

-Validate result (Quality.set, optional) assigns a quality level to a data object produced by the crowd. The task internally invokes an external validation process (custom or chosen from a library) that invokes the Quality. set API.

- Reward result (Reward.give, optional) assigns rewards to workers. Configurable rewarding logics (functions of the quality assessment values) decide for each worker whether to give or reject the reward and possibly hand the reward out.

It is important to note that the basic tactic structure is not executable as is. Rather, it lists the minimum (and typical) ingredients necessary to design a correct tactic. It is clear that this basic structure needs to be extended and turned into a correct, executable process to be applicable in practice.

\subsection{Tactics Models}

In the following, we show what it means to develop an executable tactic. We model examples of the most important tactics, namely marketplace, contest, and bid [Ipeirotis 2010; Cavallo and Jain 2012; Satzger et al. 2013] described in Section 2.2. In addition, we also model a mailing list tactic, which can be useful in CS scenarios that require targeting a precise group of people, such as for user studies.

This set of tactic models is not meant to be complete; rather, it is a set of examples of how one's own custom tactics can be implemented by the crowdsourcer. All tactics can be reused as they are or extended and adapted to new needs. We recall that a CS process may contain multiple crowd tasks, whereas each crowd task may have its own tactic. This means that within one CS process, multiple crowd tasks with different tactics may coexist, yet each crowd task has exactly one tactic.

5.3.1. Marketplace. The marketplace [Ipeirotis 2010] is one of the most common tactics for CS work. Figure 11 shows a process that represents a possible implementation. 
The model expresses the perspective of the BPMN engine inside CC. The process starts with CC loading the task definition stored in the metadata store. This operation interrogates the Task API. The task definition is used to create task instances, which are managed by the multi-instance subprocess in the figure. In the subprocess, the first operation creates the task instance, then the subprocess waits for a worker to accept the task. Upon selection by a worker, CC tries to assign the instance by first performing the possible preselection test (part of the Assign instance task): if the worker meets the requirements, the instance is started and assigned to the worker; otherwise, the instance is released and the process goes back waiting for another worker. If the instance is assigned, CC waits until the worker submits the result, which updates the task instance information through the Task API. In the case of a timeout (if a worker does not submit results within a predefined time interval), CC marks the task instance as expired and the subprocess terminates. If the task instance is instead successfully completed, the process starts the validation task, which is another subprocess. Then, the process rewards workers using the Reward API. We describe examples of validation processes and rewarding logics later.

The subprocess that manages the task instances has a timer that is triggered if instantiated tasks are not accepted or completed within a given time window. The timer triggers a task that marks all remaining instances as expired. Only if all instances are completed or expired does the process (and the CS task) end.

This is only one possible implementation of the marketplace tactic. Many others exist. For example, we can define a minimal marketplace as a marketplace with a single instance and without validation and reward. An implementation of this tactic needs to have a task to retrieve the task definition, a task to create an instance (without the subprocess), a task to assign the instance, and a task to receive the results. This corresponds to the minimal set of mandatory tasks of our basic tactic structure.

5.3.2. Contest. The contest tactic [Cavallo and Jain 2012] makes workers compete with their work for a reward. In Figure 12, we model the process of a typical contest tactic. The process is divided into two parts: the main process that is in charge of starting the task, validating results, and rewarding workers and a subprocess that manages the instantiation and execution of task instances. The subprocess is noninterrupting, which means that workers can submit results in parallel until the expiration of a deadline. Once results are collected, the process starts a validation subprocess to decide the winner(s) and then rewards the winner(s). Differently from the marketplace tactic, where the validation and reward logic are instantiated for each task instance, in a contest, they are executed only once for all instances at the same time.

The Collect results subprocess models the collection of workers' answers. It sets a timer that, after a predefined amount of time (defined by the crowdsourcer), ends the subprocess and closes the contest; this implements the deadline of the contest. Answers from workers are collected only as long as the contest is open. This behavior is modeled using an event subprocess (the dashed box) with a noninterrupting start message (the dashed start message) that can be triggered many times in parallel by different actors. The subprocess is thus executed every time an acceptance message arrives from a worker.

Various implementations exist for the contest. For instance, we can again imagine a minimal contest tactic, in which we remove the rewarding task. The other three tasks cannot be removed from the main process, as they are all mandatory. The logic of the Collect results subprocess is rather standard, and one cannot modify much. What a crowdsourcer may want to change is the logic used to decide the winner. In a minimal contest, this logic could be randomly picking a worker or selecting the first worker who submitted something. In more complex and real scenarios, this logic may require the 


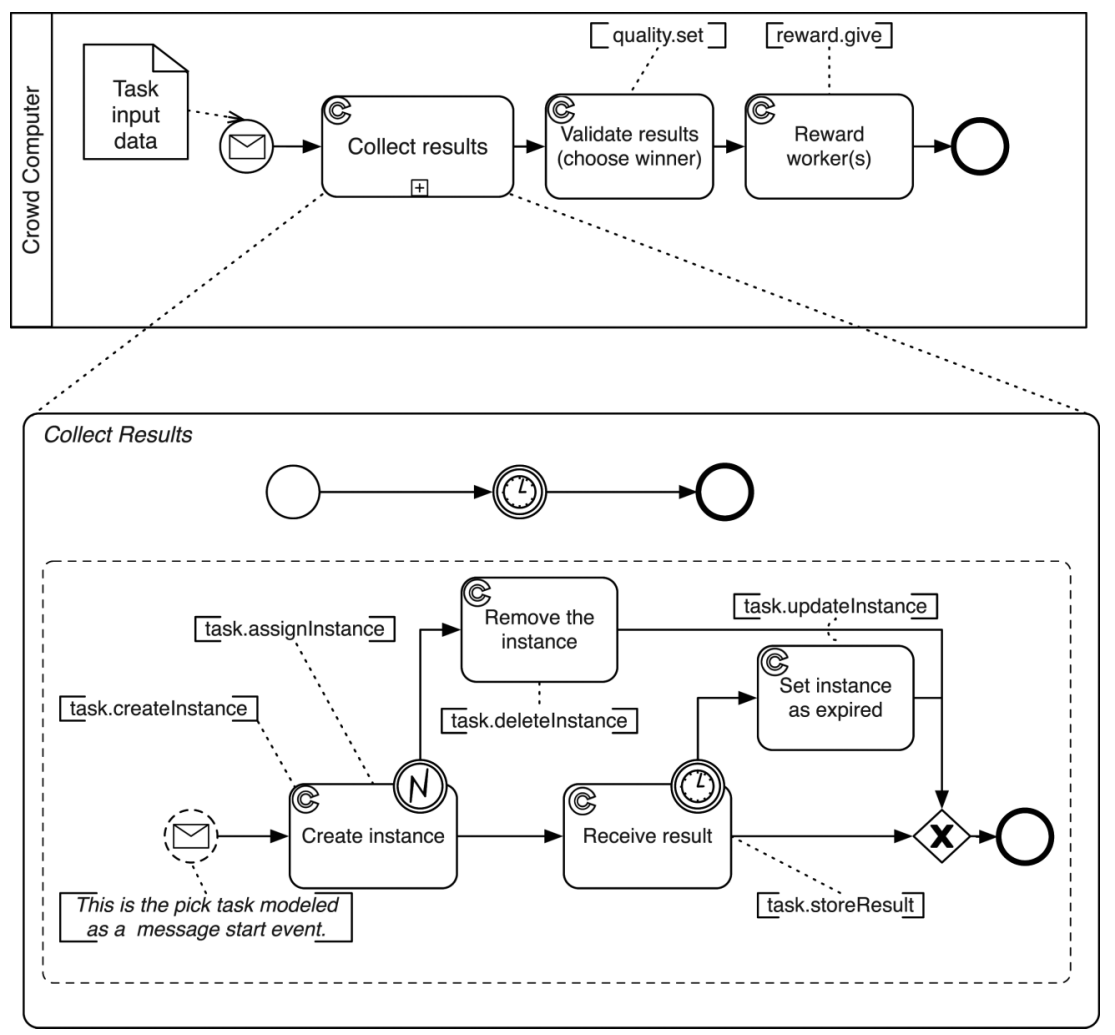

Fig. 12. Process diagram of a possible contest tactic. The main process manages the overall flow, and the subprocess manages the instantiation of tasks and the submission of results.

crowdsourcer or an external expert to select the winner, or it may ask the crowd to rank the results, or it may use a machine task to compute the winner.

5.3.3. Auction. The auction tactic [Satzger et al. 2013] fundamentally is different from the previous two tactics. Whereas in the marketplace and contest the reward is specified at the beginning, in the auction the reward is the result of a process to be run. In CS, auctions are usually executed in a reverse fashion, in which the winner is the worker who offers to perform a task at less money (the bid) than the others. The (reverse) English auction model allows workers to bid against each other for a fixed amount of time and then the lowest bid wins. In the (reverse) Dutch auction, the crowdsourcer specifies an initial reward and goes on raising it by a small amount until the first worker accepts the contract; this worker wins the auction. This type of auctions requires a significant amount of time to participate in the bid-time that is not paid and that may discourage workers from participating. Another common model is the (reverse) sealed first-price auction, in which the requester specifies the maximum amount of reward that he is willing to pay, and the first worker bidding less than this value wins. This tactic is, for example, often used by platforms with professional workers, such as programmers or freelancers.

In Figure 13, we provide an example of process diagram for a sealed first-price auction. The process shares common parts with the contest and the marketplace. From the contest, it borrows the possibility to have multiple workers competing with their bids (Check bids). The respective subprocess is therefore implemented similarly to the 


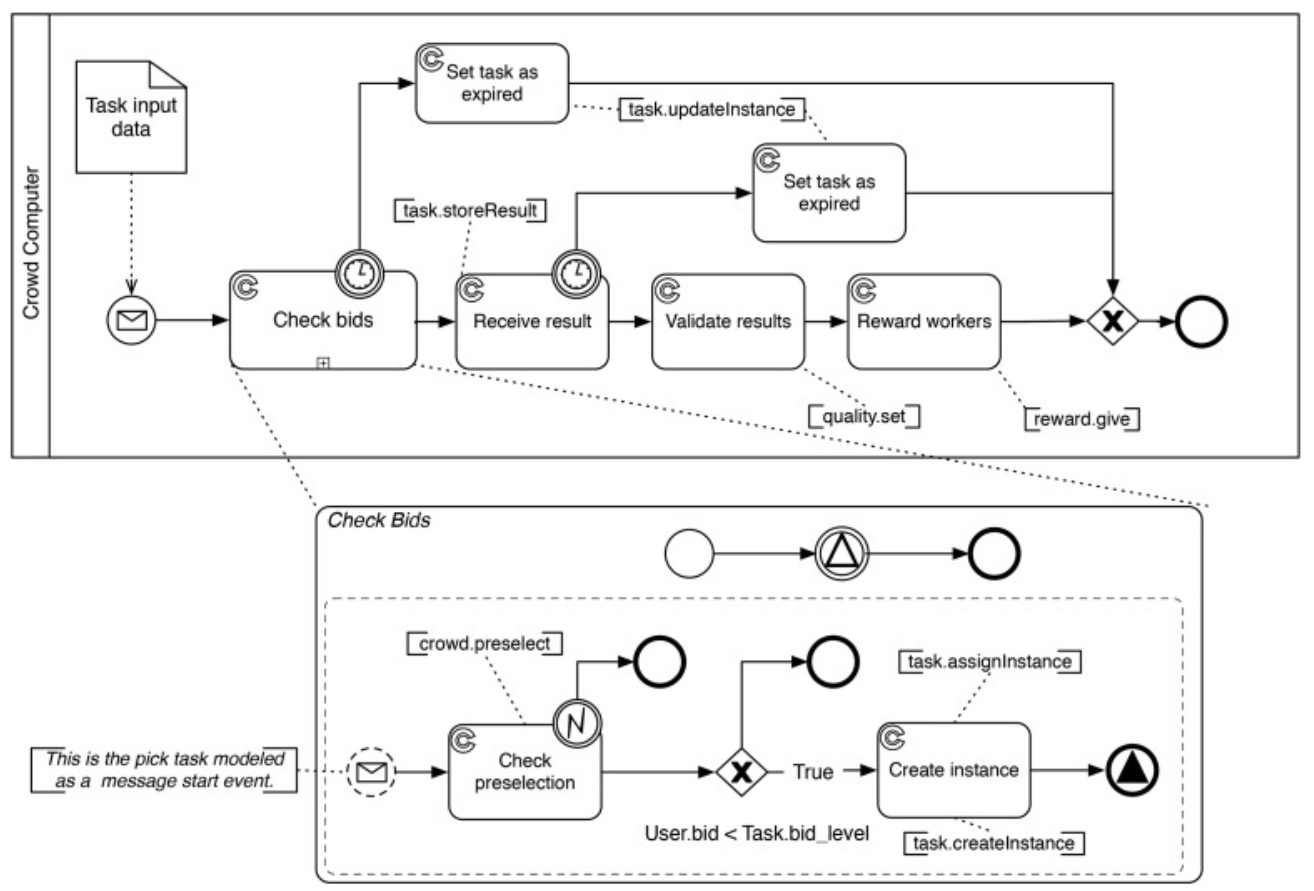

Fig. 13. Process diagram of a sealed first-price auction tactic.

one of the contest tactic, with the difference that now it is a signal (the triangle) that stops the subprocess execution. The signal is emitted as soon as a worker bids less than the threshold, and the system creates an instance of the task and assigns it to the winning worker. The main process then proceeds similarly to the marketplace tactic, yet managing only a single task instance (the one of the winner of the auction). The process ends with the usual validation of the work and the payment of the agreed-on reward based on the quality assessment.

We already explained that many other possible auction processes can be conceived. As for the minimal auction tactic, what can be eliminated is the Review result task, assuming that the worker performs satisfactorily. The reward task is mandatory, as the tactic is based on a negotiation of the reward. The bid logic (the subprocess) is most open to variations; what we modeled here is one of the simplest logics.

5.3.4. Mailing List. This tactic is not a traditional CS tactic, yet it is a common and very effective way of soliciting people to participate in some effort and get work done (e.g., questionnaires or scientific studies). The logic of this tactic is the opposite of the other tactics in that it actively pushes tasks to workers, whereas in the others, it is the workers who pull tasks. Some platforms implement similar approaches-for example, Mechanical Turk allows the crowdsourcer to assign a task to a specific person.

In Figure 14, we model the process of a possible mailing list tactic. Task instances are created automatically and assigned to selected workers in advance (Assign Tasks subprocess). The assignment keeps track of which user is assigned which instanceinformation that may be useful for validation and reward. Then the platform waits for workers' feedback, up to a given timeout. Each time a worker submits work, the system stores it, validates it, and rewards the worker. Generally, all workers are rewarded, if 


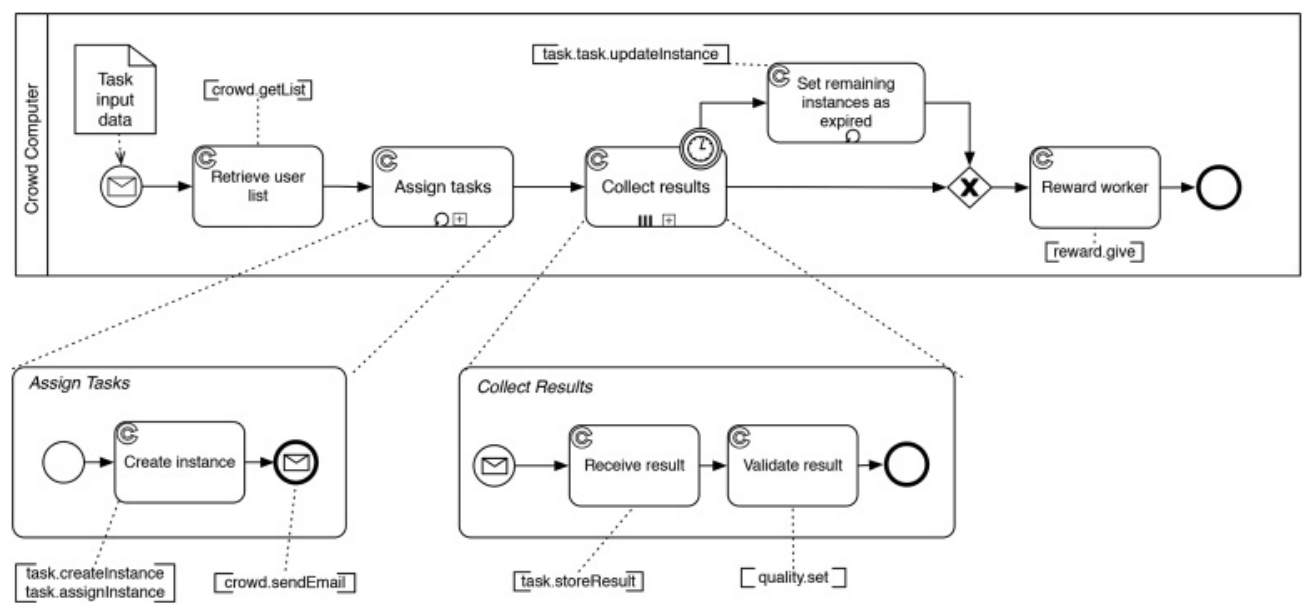

Fig. 14. Process diagram of the mailing list tactic with two subprocesses.

a reward is offered at all. If a worker is not willing to participate, the system marks the respective instances as expired after the timeout has expired.

As usual, different variations of this tactic may exist. For instance, we could anonymously invite people, such as by allowing the crowdsourcer to share a link to the task that can be forwarded via email, independently of CC. For the minimal mailing list tactic, it is enough to remove the validation and rewarding tasks.

5.3.5. Custom Tactics. The number of possible tactics to crowdsource a task-that is, the number of possible negotiation models bringing together workers and crowdsourcersis unlimited. The preceding tactics represent the most common ones that have emerged so far. Other tactics may feature moderator-based approaches, contests with groups of winners, or tactics in which the reward depends on the assessment by a control group or majority decisions [Hirth et al. 2013]. More advanced tactics may feature a twostage approach [Soleymani and Larson 2010; Hoßfeld et al. 2014b] in the assignment of tasks to workers that, for example, use one task to assess the preparation/suitability of workers and another task for the actual task of interest; access to the second task is granted only to workers considered reliable enough in the first task (pseudoreliable workers).

Sophisticated negotiation models may require complex implementations, raising the doubt whether these are still tactics or already CS processes. Technically, it is fully up to the crowdsourcer to decide how much complexity to put into the tactic and how much of it to keep outside- that is, in the CS process. For the sake of reusability across different CS processes, however, the more the negotiation logic is inside the tactic, the better. Our recommendation is to aim at tactics that allow one, at the CS process level, to focus exclusively on the integration of crowd tasks into bigger logics, possibly containing other crowd tasks, without having to focus on how to actually crowdsource work (selection of workers, quality control, rewarding).

\subsection{Validation and Rewarding Logics}

The tactic models described previously neglect the details of the last two aspects of CS: validation and rewarding. Given their reusability across tactics, the respective logics can be designed independently of the tactics and associated to them later (e.g., via simple configuration parameters). As highlighted in the basic tactic structure, validation (quality assessment) logic can be as complex as full-fledged CS processes with different 


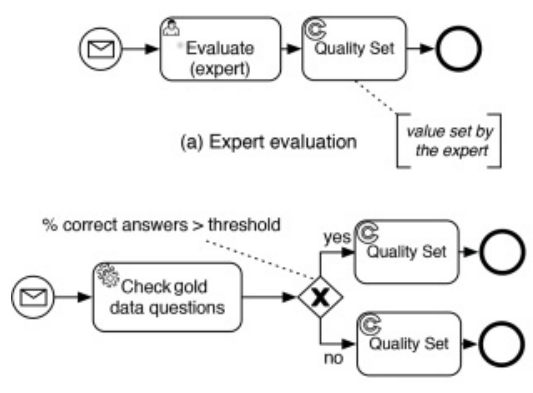

(c) Validation with automated gold data

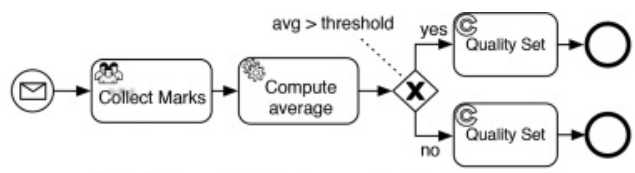

(b) Validation by letting the crowd rank

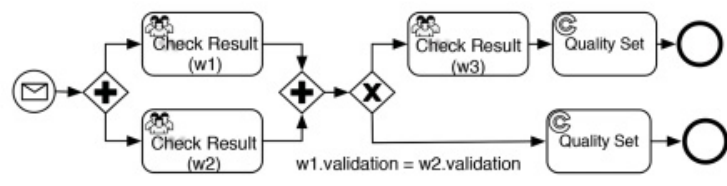

(d) Agreement by at least two out of three workers

Fig. 15. Example process diagrams of four validation processes involving different actors. All processes produce a quality assessment value in output to be stored in CC's internal metadata store.

actors involved; the rewarding logic is, instead, more of a simple configuration of how to give a reward, once quality has been assessed. For both, CC implements a set of predefined options, which we describe here. Custom validation logics can be implemented similarly by the crowdsourcer.

5.4.1. Validation Processes. Validation processes have the goal of producing a quality assessment for a given data object produced by the crowd. They are invoked by the Validate Result task of the tactics, which also stores the assessment values in CC's metadata store upon the completion of the validation processes. The most common quality assessment logics are the following (Figure 15):

(1) Expert validation: This validation involves the crowdsourcer himself or another external expert. The process consists in a simple human task that asks the expert to assess the data object and produces the assessment value as output. If the assessment is positive, the work is accepted; otherwise, it is rejected.

(2) Marking: This approach asks the crowd to mark (rank) the work of a given worker. The output is based on the average mark given by a set of workers: if such exceeds a given threshold, the work is evaluated positively and otherwise negatively.

(3) Gold data: This validation is based on so-called gold questions (whose answers are known in advance) that can be checked automatically by a suitable machine task [Oleson et al. 2011]. Checking the answers to gold questions can be used to estimate quality without analyzing the actual data objects. This validation is straightforward and does not require additional time or human activities. The percentage of correct gold answers is compared to the threshold (decided by the crowdsourcer) to decide whether to accept or reject the whole data.

(4) Agreement: This validation requires the agreement on the quality of a data object by at least two out of three judges, where judging is again crowdsourced (CS tasks). If two workers immediately agree, the agreed-on value (true or false) is chosen as output of the process. Otherwise, a third worker is asked to judge, and her judgment is used as output since it surely agrees with one of the previous two.

Various other logics can be conceived and used to assess quality. It suffices to model processes like the ones in Figure 15 (the next section provides the necessary modeling constructs and conventions), which take a data object as input and produce a respective quality assessment as output (recall Figure 10). This latter is stored as metadata and used to decide who to reward.

In this respect, it is important to understand the relationship between validation logics, tactics, and task types. The crowdsourcer chooses validation logics and tactics 
depending on (1) the task type, (2) the desired quality level of results, and (3) the available resources to spend. The use of gold data can, for instance, guarantee a good level of quality of marketplace tasks, where it is possible to automatically evaluate results against trusted data (e.g., sentiment analysis with only possible answers "positive," "neutral," and "negative"). More quality-critical tasks (e.g., nudity detection in images uploaded on children's Web sites) may require the use of an agreement logic with redundancy of answers (higher cost). Gold data and agreement can also be applied together to keep only agreed answers from a pool of workers checked against trusted data. Marking works for tasks where it is not easy to evaluate results automatically and instead a subjective evaluation is needed (e.g., voting for proposed ideas). Expert validation usually takes place in contest and auction tactics for creative tasks (e.g., logo creation). In addition, marking and expert validation can be applied together in contests, such as using markers to create a pool of top-k best results and an expert only to select among the top ones to pick the winner.

5.4.2. Rewarding Logics. Rewards are important in CS, as they motivate workers to perform tasks. The most used rewarding logics are as follows:

-All/none: This is the most trivial logic. All workers are paid (or none of them) independently of whether the work is satisfactory or not. This choice is not used often, but it can be executed automatically by a platform if no counterinstruction is given within a predefined time window.

- Upon validation: The payment upon validation is perhaps the most commonly used logic. Only the works that pass the validation step are rewarded. This type of strategy works well with tactics where workers are rewarded only if the result is satisfactory.

-The best: Here, the reward is given only to the best among all workers submitting work. This logic is generally adopted in tactics where workers compete for the reward and thus send results for a same task where only one is accepted as correct.

In addition, reward logics can be complemented with other strategies, such as the following:

-Bonus: The reward is fixed and specified when a task is created. However, workers occasionally may provide astonishing results, and a bonus reward may be given to selected workers to increment their overall reward.

-Milestones: Some tasks can require a long-lasting collaboration between the worker and the crowdsourcer (e.g., writing a software application), which can be split into milestones. In this case, the reward can be split into milestones as well. Before starting the task, the crowdsourcer and workers agree on the milestones and deadlines; at each milestone, the worker receives the corresponding reward.

With this set of reward logics, it is possible to configure most of the possible scenarios that a crowdsourcer may face. All logics make use of the quality metadata of CC and its configuration of reward payment services.

All of these rewarding logics are examples of logics already implemented in existing platforms: the all / none and upon validation logics are most commonly implemented in marketplace platforms, such as Mechanical Turk and CrowdFlower. The former can be executed automatically by the platforms and the latter only upon an explicit judgment expressed by the crowdsourcer. The the best logic is used in contest-based platforms, such as 99designs. The milestones logic is used in auction platforms, such as vWorkers, and the bonus logic is a crosscutting logic supported in several different platforms. 


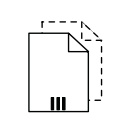

(a) Data Object Set of sets

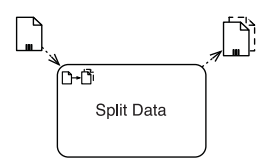

(b) Split Data Set

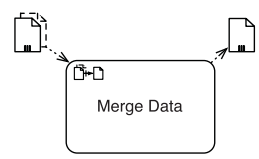

(c) Merge Data Set

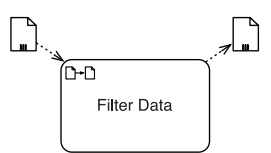

(d) Filter Data

Fig. 16. CS-specific notation for data objects and data transformations.

\section{MODELING CROWD TASKS AND DATA TRANSFORMATIONS}

With CC and the tactics, we now have almost all ingredients needed for the development of advanced CS processes. What we still need to do is clarify how to use CS tasks in practice inside CS processes and how to propagate and transform data.

\subsection{Crowd Tasks}

The core construct needed for the modeling of CS processes is the crowd task (recall Figure 1), which represents the work to be crowdsourced. The construct does not differ from a standard BPMN task only by its crowd icon but also by the fact that it actually represents a process on its own (the process of the chosen tactic) that must be properly configured before execution. A crowd task definition therefore comes with a set of parameters for crowd-related information:

-Description: Short instructions that allow workers to understand and choose tasks -Task page URL: The CS task page implementing the UI of the task

-Deadline: The date and time after which the task expires

-Tactic: The tactic process to be used as logic for the execution of the task

-Tactic parameters: Free input of custom parameters defined inside the tactic models (e.g., 'NumberOfInstances $=10$ ')

-Reward: The amount of the reward for the task (e.g., \$10)

-Reward strategy: The strategy of the Reward Worker task (e.g., the best)

-Preselection condition: Boolean expression over user profile parameters (e.g., User.Gender = 'Woman' AND User.Language = 'English')

The CS and the orchestrating of work inside a CS process is thus as simple as setting these properties for each crowd task of the process. However, the CS of work via multiple crowd tasks may ask for proper data transformation logics.

\subsection{Data Transformations}

Crowd tasks usually ask workers either to produce some output that can be inspected (e.g., photos or text) without input from the crowdsourcer or produce some output (e.g., tags or translations) given a dataset provided as input (e.g., photos or text). It is also common that consumed and produced datasets are large and that crowd tasks work with different input/output dataset sizes. For example, one task may ask workers to upload one photo, whereas another task may ask them to compare two photos or select the best out of three given photos. Input dataset sizes are usually fixed; output dataset sizes may also be variable (e.g., a task may allow workers to upload a generic set of photos). The mismatch that may arise between output and input dataset sizes asks for proper data management operations.

BPMN has native constructs to specify data objects and associations of data objects with tasks, with the association arrows specifying the dataflow of the process. Data objects model individual objects (see Figure 9) or collections of objects (if marked with the multi-instance label-that is, three vertical lines). To model collections of collections, in Figure 16(a) we define the set of sets data object. This allows us to model, for example, 10 sets of three photos each to be crowdsourced using 10 instances of a given 


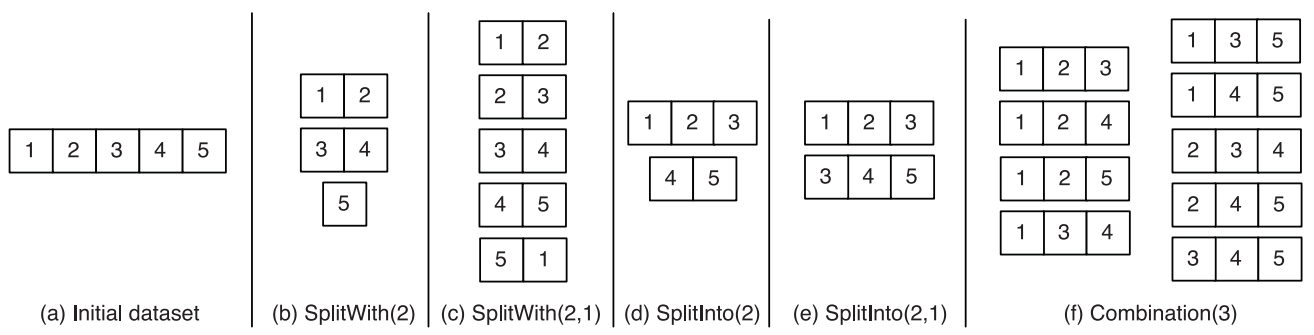

Fig. 17. The different uses of the dataset splitting operator of BPMN4Crowd.

crowd task. Properly splitting, merging, and filtering datasets further requires a set of dedicated operators; Figure 16 shows their constructs.

6.2.1. Splitting Datasets. The Split Data operation allows splitting datasets into subsets. It takes as input a set of data objects and produces as output a set of sets. The operation can be configured to use different splitting logics, as illustrated in Figure 17:

-SplitWith(n,r?): This function allows the crowdsourcer to specify the size of the output sets; the number of produced sets depends on the size of the input dataset. The optional parameter $r$ specifies redundancy: if set, consecutive datasets will contain $r$ similar data objects. For instance, the operation is used when the crowdsourcer wants to assign to each task instance a precise number of input data objects without worrying about how many task instances need to be created.

-SplitInto(n,r?): This function splits the input dataset into $n$ subsets of similar size. The optional parameter $r$ specifies redundancy: if set, consecutive datasets will contain $r$ similar data objects. The operation is used when the crowdsourcer wants to control how many task instances will be created (one instance per subset).

- Combination( $n$ ): This function generates all possible combinations of size $n$ from a given dataset in input. Combinations are redundant in that they share repeated elements. They provide for maximum redundancy with the minimum number of output datasets.

6.2.2. Merging Datasets. The opposite of splitting datasets into subsets is merging a set of subsets into an integrated dataset. BPMN4Crowd uses the Merge Data operation for this purpose. The merging logic is unique and works at the data object level (it takes all elements without distinctions), and no further configuration is required. It takes as input a set of sets and produces a set of items. The operation can be used to recompose the results of different task instances into a unique set of results.

6.2.3. Filtering Data Objects. Finally, the Filter Data operation allows the crowdsourcer to filter data objects inside datasets. Filter conditions can be specified over the metadata (e.g., data properties or quality assessments) associated with the data objects in CC's metadata store. Only data items that match the filter condition pass the filter. For example, a filter can be used to filter out those data objects that have been evaluated positively by the respective quality assessment process.

6.2.4. Modeling Example. We are now ready to refine the initial logo assessment example described in Figure 4. Figure 18 illustrates the BPMN4Crowd model of the process with the necessary data transformation operations in place. We recall that the crowd task (Evaluate logo) asks workers to assess the quality of a logo, which as a result produces a collection of marks. We assume that for this purpose we implement a task page that shows the logo together with a form with a set of HTML radio buttons that allow the worker to express her mark. Each task instance thus requires the logo as 


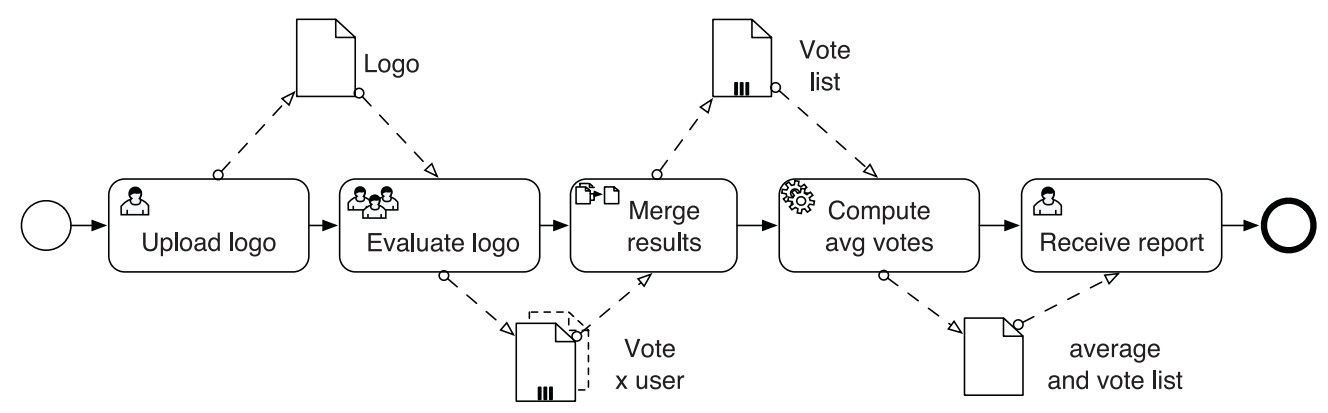

Fig. 18. The CS process of the photo contest scenario modeled with BPMN4Crowd.

input and produces one mark as output. The logo is supplied by the requester uploading it in the first task. To compute the average, we merge the marks into a unique list (Merge results task) and use a machine task (executed internally by the process engine, Compute avg votes) for the math. The average value can then be inspected by the crowdsourcer via the process interface (last human task). The tactic of the crowd task does not require the crowdsourcer to validate results and automatically gives, for example, $\$ 0.05$ to all workers.

\section{IMPLEMENTATION}

To ease the creation and execution of BPMN4Crowd CS processes, we implemented three tools:

-A BPMN4Crowd editor to support the graphical design of CS processes (http://editor.crowdcomputer.org); the editor is based on the Activiti BPMN modeling tool (http://www.activiti.org).

-A BPMN4Crowd compiler to transform process models into executable BPMN processes and configurations for CC, as well as a process deployer able to deploy CS processes onto CC (http://compiler.crowdcomputer.org).

-An extended BPMN engine (based on the Activiti engine) that contains the library of CC APIs that enable the communication between the engine and CC (http:// engine.crowdcomputer.org).

Next we present how we implemented the three tools.

\subsection{BPMN4Crowd Editor}

Given the intrinsic complexity of process editors, for the implementation of the BPMN4Crowd editor we opted for the extension of the well-known Activiti Designer, a BPMN plugin for Eclipse (http://www.eclipse.org). The tool supports the implementation of custom tasks, whereas it lacks support for other custom artifacts, such as new data object constructs, and it does not implement all features of the standard BPMN specification-for example, it does not support the noninterrupting event subprocesses (useful for the tactics) nor the message end event (useful for the validation processes). For this reason, the current implementation of the BPMN4Crowd editor provides some of the BPMN4Crowd modeling features differently than presented before: extended data objects (sets and sets of sets) are not specified using data object artifacts connected to tasks, but instead we use task parameters to declare data objects and specify dataflows. CS tactics and quality validation logics can be modeled as independent processes that can be assigned to crowd tasks and configured via suitable task parameters. 


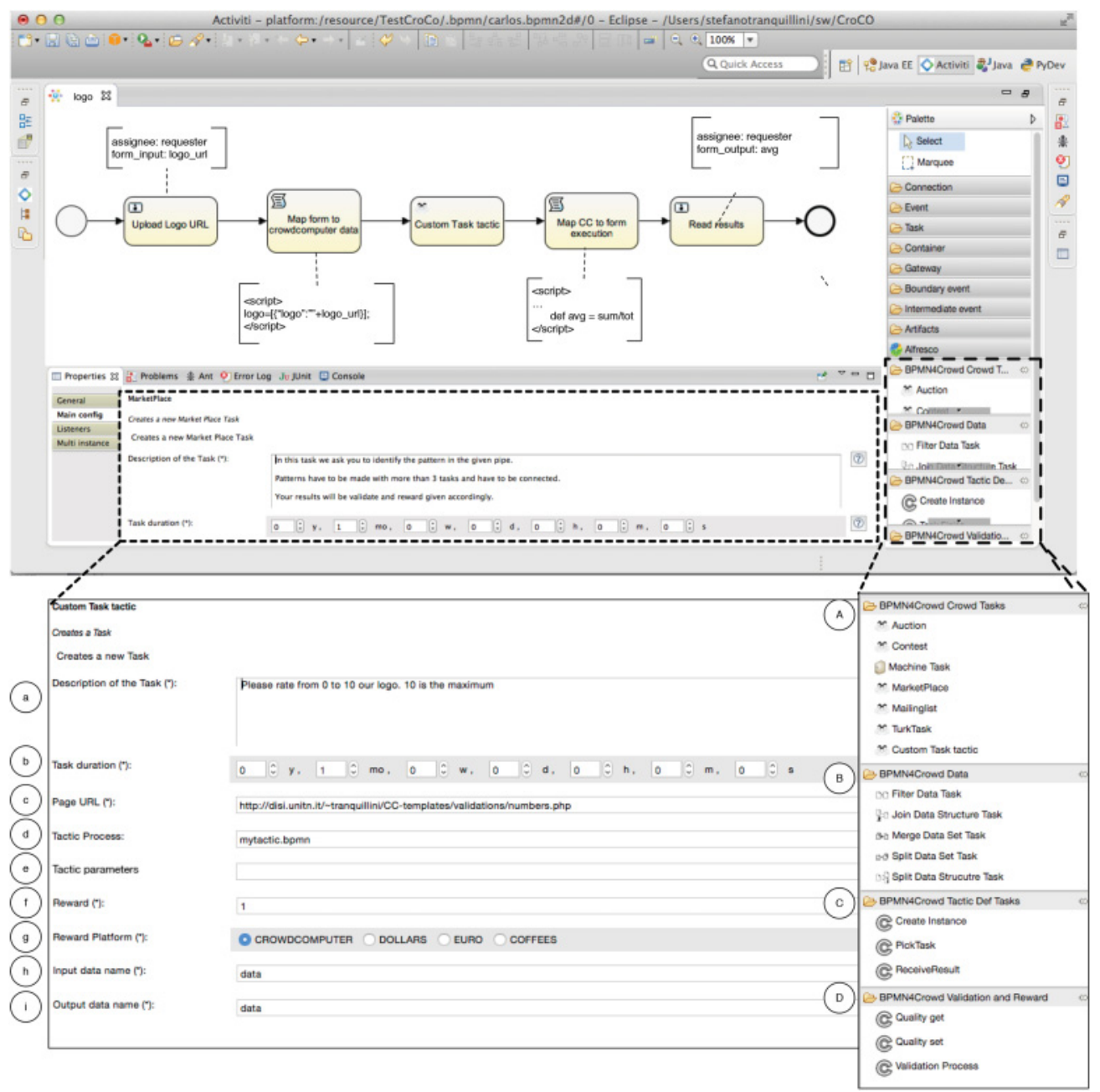

Fig. 19. The visual BPMN4Crowd editor in action. The figure highlights the BPMN4Crowd palette with new modeling constructs and the properties window for the setting of task parameters.

With the BPMN4Crowd editor, a crowdsourcer can easily create his own CS processes. Figure 19 shows a screenshot of the editor. On the right-hand side of the figure, there is the palette that contains the crowd tasks (A), data transformation tasks (B), and CC API tasks to define the tactic (C) and define the validation process (D). The crowd tasks (A) enable the reuse of predefined tactics (the ones presented in Section 5.3) and custom tactics; TurkTask also allows posting tasks on Mechanical Turk. In addition, we added a machine task, different from standard BPMN, to ease the integration of external Web services with CC. The machine task can be used to get data that are used in the process (load data) or post data to execute external services. Each data transformation task (B) implements a specific data operation (e.g., merge or split). The current implementation also supports a prototypical implementation of split and merge operations on metadata structures. For CC API tasks (C and D), we implemented a set of dedicated tasks that wrap specific API calls. In (C), we have all tasks that can be used to model a custom task. In (D), the modeler has the tasks that are used to call 
and model a validation process. The Quality set task allows invoking the Quality. set CC operation directly from within a validation process.

The central part the BPMN4Crowd editor features the canvas where the process is modeled. The crowdsourcer can drag and drop tasks from the palette and compose his own CS process. The view in Figure 19 is the process model for the logo assessment example introduced in Figure 4. For each data transformation or crowd task, the crowdsourcer can specify a set of configuration parameters in the property tab in the lower part of the editor. The parameters are the ones already specified for crowd tasks (Section 6.1), plus additional fields that are used to specify input and output data objects as well as others that refer to the configuration of the tactic, such as the tactic process and reward parameters:

(1) Description of the task: This is the text that is presented to the worker when she starts the task. It should describe what the worker has to do to complete the task.

(2) Task duration: This parameter specifies for how long the task will be active. After this time, the task will automatically be stopped by the system (deadline).

(3) Page URL: This is the parameter that specifies the URL of the task page created by the crowdsourcer. The task page implements the interface of the crowd tasks.

(4) Tactic process: Since each custom crowd task has its own tactic process, here the crowdsourcer can specify the process name of the tactic process to adopt.

(5) Tactic parameters: Here the crowdsourcer can pass configuration data from the CS process to the tactic process.

(6) Reward: This parameter specifies the quantity of the reward to be given to workers whose work is evaluated of sufficient quality.

(7) Reward platform: CC has a plugin interface able to support various types of payments. With this parameter, the crowdsourcer is able to decide which platform (e.g., PayPal) to use to reward workers.

(8) Input data name: This parameter is used to specify the name of the data object from which to read data as input.

(9) Output data name: This parameter specifies the name of the data object to which to write output data.

These implementation conventions assure the full expressive power of BPMN4Crowd as introduced in this article.

\subsection{BPMN4Crowd Compiler and Deployer}

BPMN4Crowd contains both standard BPMN constructs and instructions for CC APIs and task pages. This makes the language not immediately executable by a standard BPMN engine. The compiler therefore takes as input a CS process, rewrites parts of it, and creates a zip file that contains executable BPMN processes (the CS process and all of its validation processes) that can be deployed on the engine.

The compiler modifies the process in five key aspects, graphically illustrated in Figure 20, which shows the differences between compiled and noncompiled processes: Crowd tasks, Pick tasks, Receive tasks, Validation tasks, and the end event in tactic models. The compiler adds a receive message after each crowd task to receive a notification of task completion from $\mathrm{CC}$ front-end upon termination of the respective tactic process. A similar compilation is repeated for all validation processes linked by the crowd tasks of the process, adding a receive task after each Validation task. For the internals of the tactic processes, the compiler converts the Pick and Receive tasks into message receive tasks, if these are not yet present (e.g., this is used by the tactics in Figures 12 and 13), and adds a Task Finish task before the end event of tactic models (a system operation that closes the tactic). 


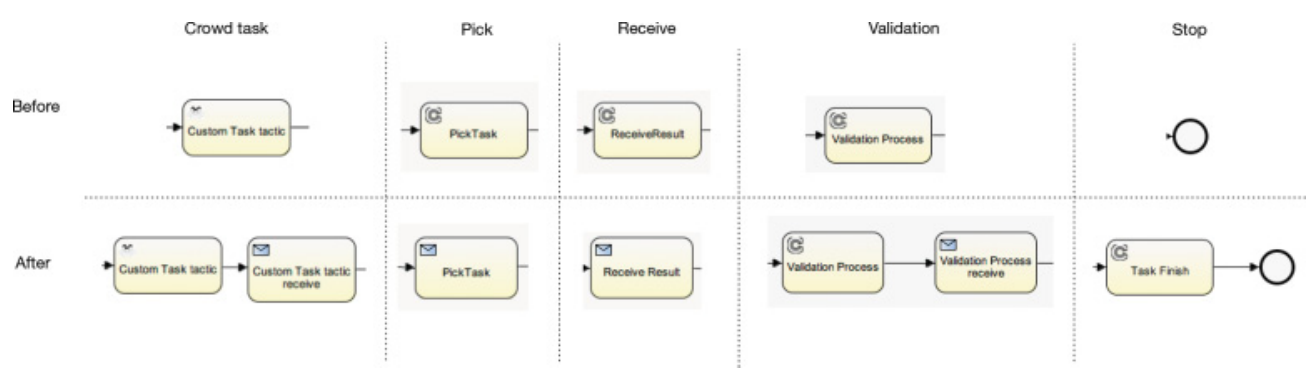

Fig. 20. The transformation of the processes before and after the compilation step.

Once the compilation is finished, the crowdsourcer can take the zip file and upload it to the deployer that unzips the file, extracts crowd-related information, creates the necessary data structures and metadata to handle the execution of crowd tasks, and deploys the process on the BPMN engine. Then, the process can be executed.

\subsection{Extended BPMN Engine}

Upon deployment of a process, the start message of the process is sent by the deployer to the BPMN engine to start execution. Standard BPMN constructs are processed according to their standard semantics. To handle the execution of a crowd task on CC, we extended the engine with additional logic (Java classes). For every crowd task, there is a Java class that sends to the task manager API of CC the task configuration parameters (specified in the process model) and runtime data objects, such as the output of a previous task. The task manager receives the data and instantiates the corresponding crowd task by instantiating the respective tactic process. For each new crowd task, the task manager creates its own tactic instance and makes the task available to workers. Workers are then able to execute the task instances and send results. Workers' results are sent via CC API to the task manager, which updates the metadata of the corresponding instances. Afterward, the task manager executes the validation process. This process is again implemented as a BPMN process. After the validation, the task manager executes the reward procedure. This is a function of the reward manager, which executes the logic specified by the crowdsourcer and updates the metadata relative to rewarding, giving the reward to the workers. Once the tactic execution is completed, the task manager gives back the execution control to the BPMN engine, sending the task metadata resulting from the execution as well.

Data tasks are executed in a similar fashion. We created dedicated APIs for each operation (we do not show these in the Figure 6 because it is a mere implementation choice). The Data API implements the operations of the functions discussed previously. Their execution is straightforward: the APIs accept as input data, execute the selected operation, and return the result to the BPMN engine in the form of a message that contains the metadata results.

\section{CASE STUDY}

In the following, we discuss the benefits and limitations of BPMN4Crowd and the described CC prototype if leveraged for the implementation of a concrete CS process that we carried out at the University of Trento in the context of another research project: crowd-based pattern mining.

\subsection{Goal and Requirements}

Harvesting knowledge from large datasets (i.e., data mining) is a domain where computers generally outperform humans, especially if the dataset to be analyzed is 
large. If the dataset is instead small and the knowledge to be identified is complex (e.g., model patterns), humans may outperform machines. Starting from this observation, in Rodriguez et al. [2013, 2014], we started investigating whether it is possible to use the crowd to mine patterns from small datasets; we specifically focused on the domain of mashup model patterns to feed our pattern recommender Baya [Roy Chowdhury et al. 2011, 2012] implemented for the data mashup tool Yahoo! Pipes (https://pipes.yahoo.com).

Mashups [Daniel and Matera 2014] are composite Web applications developed starting from reusable data, application logic, and/or UIs sourced from the Web. Mashup tools, such as Yahoo! Pipes, aim to ease the development of mashups, most commonly via model-driven development paradigms that produce models that can be parsed or compiled for execution. Models hence reflect mashup development knowledge, and model patterns (reusable, modular model fragments) may help share and reuse such knowledge. A typical pattern (see, e.g., Figure 22) contains a set of components (e.g., RSS feeds or data processing logics), connectors (propagating data among components), selections, and configurations (e.g., data inputs). The video of how Baya works in practice (https://www.youtube.com/watch?v=AL0i4ONCUmQ) provides a good feeling of the kind of models that we target and their benefit in the development of data mashups (mashups processing data sourced from the Web).

The experiments carried out were designed, implemented, and coordinated manually, although as a matter of fact, the methodology followed can be seen as a CS process involving human actors (researchers), machine tasks (e.g., to compute metrics), and a set of crowd tasks. In the following, we show how CC and BPMN4Crowd can be used to implement the same experiments as a CS process.

We summarize the requirements of the study as follows:

-Crowd algorithms: We would like to compare three different "crowd algorithms" for pattern mining - that is, three task implementations asking workers to identify patterns with different levels of complexity and information available:

-Naive shows individual models to workers and asks them to identify pieces of reusable knowledge - that is, patterns;

-Random 3 presents three different models and asks them to identify patterns that recur in at least two of the models; and

- ChooseN allows workers themselves to choose $\mathrm{N}$ models from a set of 10 given models and asks them to identify patterns inside the $\mathrm{N}$ models.

-Research question 1: After running the three algorithms, we would like to compare the respective patterns produced by computing a set of simple metrics, such as the number of patterns produced, the average size of the patterns, and the cost per pattern. This is done to be able to choose the "best" algorithm (if any) and set of patterns for the next step of the study.

-Quality assessment: Data mining commonly requires an expert to validate discovered knowledge. In the context of mashup model pattern, we are especially interested in studying the understandability, usefulness, reusability, and novelty of patterns, and we would like to study whether this validation can be crowdsourced as well by implementing two different validation "algorithms" to be compared:

-Expert assessment is done manually by ourselves (experts in the field). This assessment serves as ground truth for the comparison.

- Crowd assessment is done by the workers and represents the object of the study. The task is the same as the one for the experts.

-Research question 2: Given the two assessments, we would like to take a decision on the applicability of CS for the validation of mined model patterns.

The CS logics of the two dependent studies can be abstracted as a configurable process: 


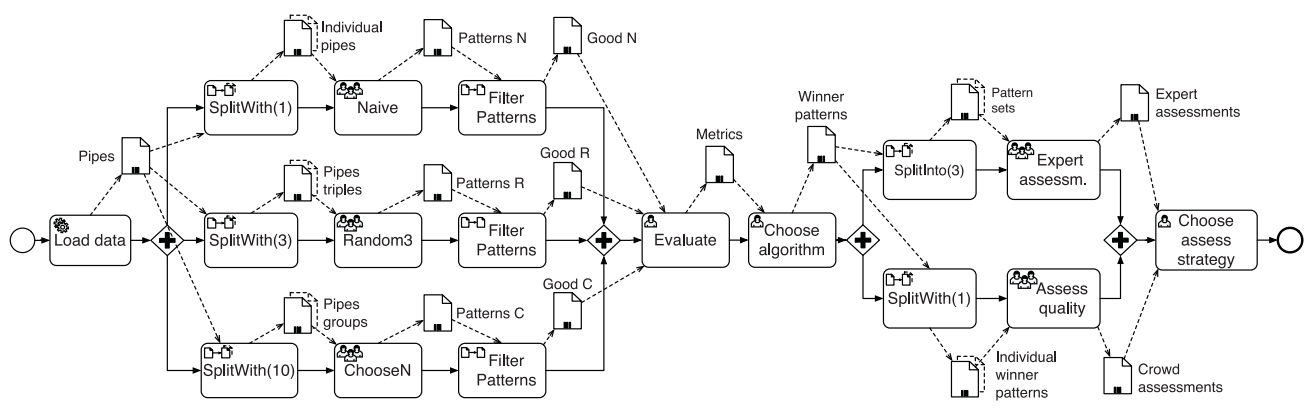

Fig. 21. The CS process of the crowd-based pattern mining scenario. All except one crowd task use a marketplace tactic; the Expert Assessment task uses a mailing list tactic for predefined experts. In line with our prototype implementation, we omit the Data Merge task after the crowd tasks.

(1) Load mashup models and initialize dataset: To ease the work of the crowd, we use screenshots of the models as rendered in Yahoo! Pipes as the dataset.

(2) Partition dataset and map to tasks: The three mining tasks require 1,3 , and 10 models as input per task instance; the validation tasks requires a third of the dataset per expert (three researchers are involved) and 1 model per crowd task instance.

(3) Deploy tasks: The tasks must be deployed on CC and made available to the crowd and experts.

(4) Manage task execution and collect patterns: Tasks must be executed by the crowd and experts, and patterns must be collected by CC.

(5) Integrate results: Results from workers must be integrated into an output dataset, possibly filtering out results of workers with poor performance (based on control questions).

For presentation purposes, we keep the requirements and descriptions here simple; the complete details can be inspected in the references cited in this section. For instance, we omit the details of the control questions used to assess the performance of workers or the input fields added to the tasks to collect additional metadata.

\subsection{Process Model}

Figure 21 illustrates a possible BPMN4Crowd process model implementing the whole study in an integrated fashion. The process starts with loading the screenshots of the models (also called pipes in Yahoo! Pipes) from a prefilled repository. Then, the process proceeds with three parallel branches, one for each crowd mining approach to study. The Naive branch splits the dataset into subsets of size 1 using the SplitWith(n) operator; the Random 3 and ChooseN branches do the same with subset sizes of 3 and 10. Next, the actual mining is crowdsourced via three marketplace crowd tasks, each followed by a Filter operator dropping patterns that have fewer than two components selected, are not connected, and do not have all input fields filled. Then, all collected patterns are used by a researcher to compute a set of metrics (number of patterns, average pattern size, cost per pattern) that, in turn, are used by a researcher to choose the algorithm with the best performance.

Starting from the set of patterns of the chosen algorithm, the process proceeds with the study of whether the crowd can be used to validate patterns. It splits the patterns into three equal subsets to be validated by the three experts and subsets of size 1 for the workers. The process models the involvement of the experts as a crowd task, in that it uses a mailing list tactic to assign the tasks to them. The crowd assessment is 
Name and description of pipe sources from Yahoo! Pipes
The pipe model to be analyzed by the worker. The model is a clickable image map that allows the worker to define a pattern by selecting its components.

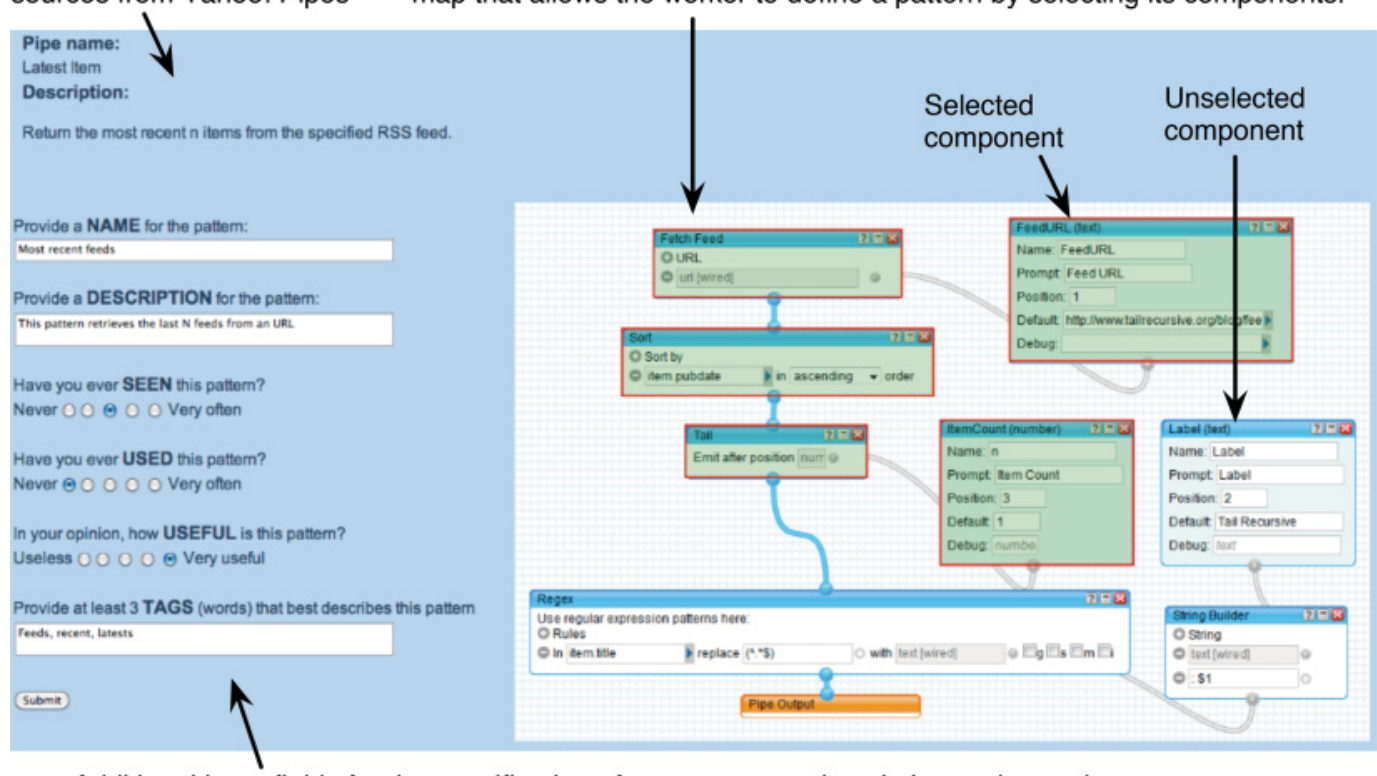

Additional input fields for the specification of pattern name, description and metadata

Fig. 22. Task design for the selection, description, and rating of mashup model patterns.

instead again performed using a marketplace tactic. The process ends with a researcher comparing the results manually and making a final decision on the research questions.

\subsection{Implementation}

Figures 22 and 23 are screenshots of the task pages implemented to crowdsource the Naive pattern identification task and the pattern validation tasks (for both experts and the crowd), respectively; the screenshots of the Random3 and ChooseN tasks can be found in Rodriguez et al. [2013].

Given these task implementations, the modeling of the process in Figure 21 inside the BPMN4Crowd editor is straightforward. We replicated the logic within the editor, configuring each task according to the conventions explained in the previous section. The resulting process model is shown in Figure 24, in which, for better readability, we graphically annotate tasks with the parameters that must be specified as task properties in the editor. To model the process, we decided to use the crowd task with predefined tactics. The figure shows that the implemented editor and runtime environment support all necessary features described previously in this article.

Figure 19 shows the editor with the first part of the process in the modeling canvas along with the details of the parameter settings for the Naive crowd task. Specifically, the Naive crowd task uses the marketplace tactic illustrated in Figure 11 with an evaluation of the results based on gold data, as presented in Figure 15(c). The reward of the task is $\$ 0.50$ upon successful validation. The description of the task tells the goal and rules of the task. The deadline is set to 1 month. The page URL, the interface of the task, points to the page shown in Figure 22. The input data object is set to "Individual pipes," and the output data object is set to "Patterns N." The other crowd tasks are configured similarly. The figure also annotates data transformation tasks with their configurations. For example, in the first branch, the Split task is configured to divide the set of models into sets of one element each. 


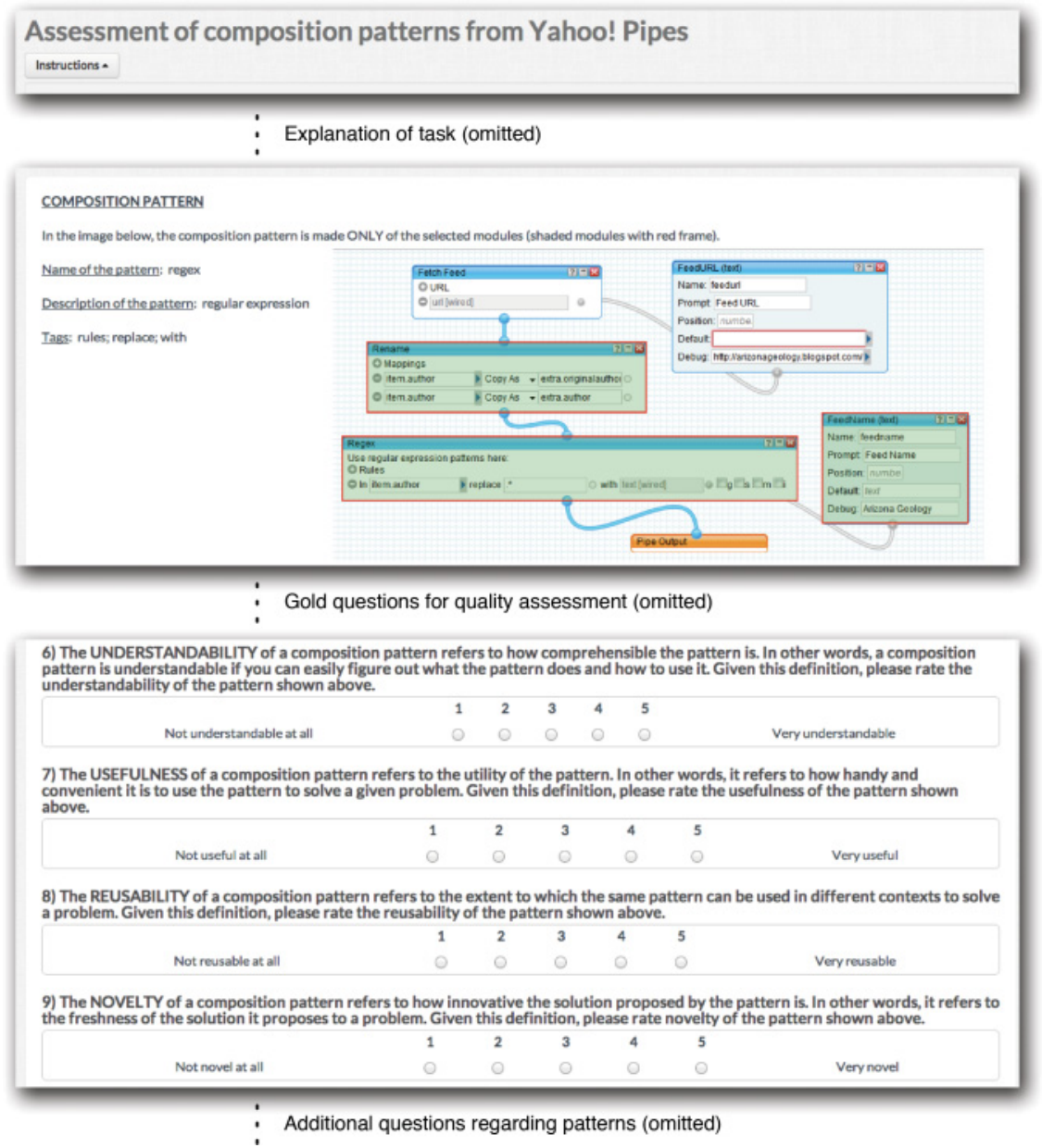

Fig. 23. Task design for the validation of collected mashup model patterns. We omit the initial description, gold questions for worker assessment, and additional inputs.

All crowd tasks are implemented with standard Web technologies (HTML, CSS, JavaScript, and Java on the server side) and deployed on a Web server hosted by Amazon Web Services. Mashup models are identified (the metadata exchanged between the task pages and CC) using the filenames of the models as stored on the Web server. The human and machine tasks are standard BPMN constructs (assigned to ourselves and an initial pattern loading Web service) and, as such, are managed directly by the Activiti BPMN engine, which can be deployed on a common Web server. Activiti natively exposes a set of APIs that CC can use to deploy, execute, and control the process.

\subsection{Analysis}

The outlined implementation shows how BPMN4Crowd eases both the design and the conduct of the described study. For the actual study described in Rodriguez et al. [2013], we did not yet have CC and BPMN4Crowd and therefore had to implement everything from scratch and by hand (so far, we only studied the first part of the algorithm, i.e., the comparison of the three pattern mining tasks). For instance, we developed three separate Web applications, one for each pattern mining task, each 


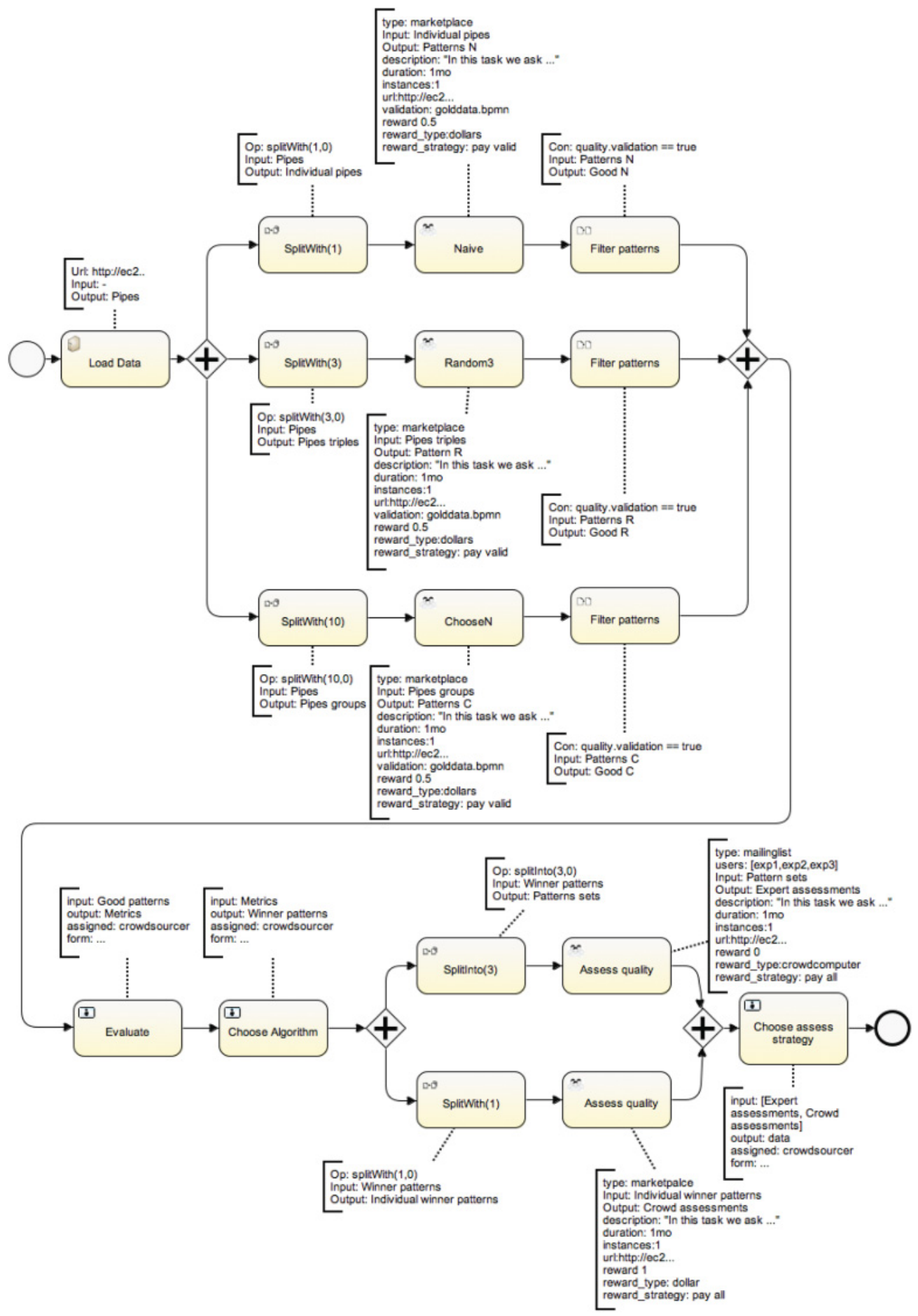

Fig. 24. Implementation and configuration of the CS process of Figure 21 in the BPMN4Crowd editor. Annotations correspond to task configurations and make them explicit in the model. 
comprising its own data store, data management logic, task UI, data task instance assignment logic, and pattern validation logic. Figure 22 is the screenshot of the original task page used. We manually configured the database underlying the applications and implemented the logic to load the pipes dataset both in JSON (for all pipes extracted from the "most popular pipes" category) and PNG (for the sample used to crowdsource the task). We implemented the algorithms (one for each application) to split the dataset as needed and manually mapped each partition to a task instance. We manually created as many task instances as we had data partitions, so as to have a task instance, thus a worker, for each partition. As a concrete CS platform, we used CrowdFlower (www.crowdflower.com), which features a marketplace tactic with gold data for worker assessment. For each crowd task, we created a task on CrowdFlower that contained the questionnaire with the gold questions to evaluate workers' expertise, plus a link to our Web applications (the task pages) where workers could perform the pattern identification tasks. Gold data were used as discriminant to accept or reject results, filter out bad results, and reward workers. The filtering was done manually: data were downloaded from CrowdFlower and from our Web applications and were matched and filtered according to the criteria outlined previously. In the same filtering operation, we also checked if the patterns met the requirements of what we called good patterns (minimum two components, connected model, meaningful text inputs), eliminating the ones that did not. The three CS experiments were run sequentially, first Naive, then Random3, then ChooseN. When all three experiments were completed and all data were integrated (manually), we compared the results and decided which was the best approach to mine model patterns with the crowd. For curiosity, it turned out that the Naive approach produced the most patterns, with good variability in terms of pattern sizes and at the lowest overall cost (after applying all checks) [Rodriguez et al. 2013]. In Rodriguez et al. [2014], we further compared the performance of Naive against that of an automated mining algorithm, obtaining similar results.

The BPMN4Crowd process model in Figure 24 shows well how much of the described manual effort can instead be automated with CC. The two approaches are both effective but require a significantly different amount of time for their development. Of course, the implementations of the individual task pages must still be done manually by the crowdsourcer. However, the respective Web applications no longer need to take care of managing task instances and data task instance assignments, as this is now done by CC. What is needed instead is the exchange of the respective metadata with CC, which is a minor effort compared to the full implementation of the data management and task instantiation logics. Given the previous implementation of these task pages, their reuse in CC is straightforward. The Load Data task that fetches the pipes models from the local repository can be implemented as a common Web service.

Given these implementations, the use of CC and BPMN4Crowd allows one to automate the management and execution of 14 tasks out of the 17 tasks in Figure 24 (next to the control flow and propagation of data): the machine task to load data is executed automatically by calling the respective Web service; the eight data transformation tasks are done inside CC, and the five crowd tasks (including the expert assessment task) are deployed, executed, and integrated automatically. What is left as pure manual work are the three human tasks, which require the direct involvement of the researchers (in addition to the involvement into the expert evaluation, which is part of the study design and not attributable to the coordination of the study). In addition, the availability of the model of the process and the possibility to easily change parameters of tasks or to reconfigure the model enables researchers to fine-tune the process and rerun experiments without spending additional time for coding or coordinating tasks. 


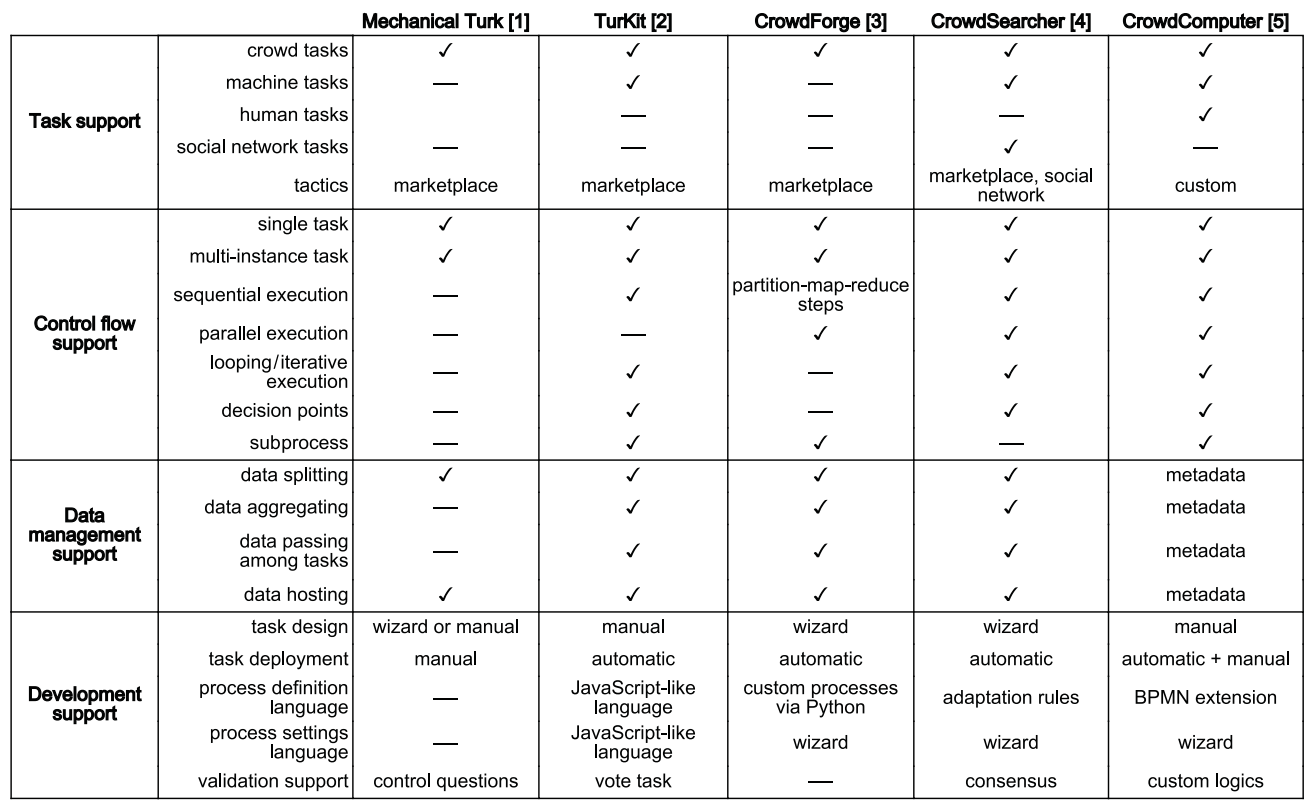

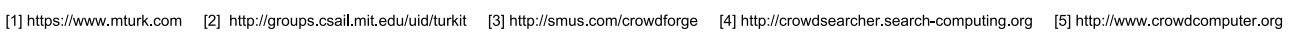

Fig. 25. Comparison of the development support provided by different CS platforms.

\section{COMPARATIVE ANALYSIS AND LIMITATIONS}

The target users of BPMN4Crowd are process modelers who want to crowdsource work in a fashion that is integrated with their BPM practices. Today, BPM typically involves the design and execution of process models expressed in BPMN. The possibility to intermix tasks executed by a crowd with tasks executed by other actors, such as the crowdsourcer herself or a Web service, by means of an extension of BPMN makes the creation of integrated and structured CS processes feasible. Compared to state-of-theart CS platforms, the binomial CC/BPMN4Crowd provides for this integration in a natural fashion. We thus consider the approach described in this article particularly effective and powerful in cases where the outlined kind of integration is needed.

In this respect, it is important to realize that advanced CS scenarios generally are not trivial and require good domain knowledge by the process modeler, as well as working knowledge of CS. CC and BPMN4Crowd both enable new features (e.g., tactics) and simplify their use (e.g., via suitable abstractions and repositories of reusable models); however, the intrinsic, conceptual complexity of advanced CS processes cannot be eliminated. This is true also for other CS approaches that target similar composite CS processes.

In Figure 25, we compare CC and BPMN4Crowd with a basic marketplace CS platform (Mechanical Turk) and three other platforms for the management of complex CS processes (TurKit, CrowdForge, and CrowdSearcher-see the next section for detailed descriptions). The requirement for the selection of these platforms is either their free accessibility online (Mechanical Turk, CrowdSearcher) or the availability of their source code (TurKit, CrowdForge). The comparison is based on a selection of features that impact the complexity of developing CS processes; we group the features into task support, control flow support, data management support, and development support features. The more the features are supported by a platform, the more the platform 
eases the development of processes that require these features and the less manual effort is needed.

$\mathrm{CC}$ is the only platform that supports human tasks-that is, integration with conventional BPM practice-whereas CrowdSearcher comes with support for tasks performed on social networks (Facebook). CC is the only platform that supports custom CS tactics, and thanks to the use of BPMN, it supports all control flow constructs. It also supports custom validation logics, whereas other approaches typically support predefined validation logics, if any. One distinguishing feature is that CC manages metadata, not actual data, and the crowdsourcer decides where to store the data on his own. This choice reduces the amount of data transferred over the network and allows the crowdsourcer to protect data that are sensitive (e.g., images with nudity) or subject to local regulations (e.g., healthcare data of citizens in some countries must be stored on country-local servers).

The previous comparison shows that for simple or individual crowd tasks not requiring custom logics or having special data management requirements, CC can be used but may result in more complexity than necessary. To crowdsource a single crowd task, a conventional CS platform (e.g., Mechanical Turk) may be easier to use and manage. To crowdsource a crowd task that is of pure data processing nature, a dedicated instrument like CrowdForge or CrowdDB [Franklin et al. 2011] may be more efficient. However, if the crowd task is to be integrated with existing legacy business processes, $\mathrm{CC}$ may again be more efficient.

The choice of extending an existing process modeling language and related technologies has a threefold benefit (next to the native support for the control flow features): (1) it can foster the adoption of BPMN4Crowd, as BPMN is widely known and adopted to model business processes; (2) people who already use BPMN can easily integrate and extend their processes with tasks executed by the crowd; and (3) the implementation of the necessary runtime environment can rely on existing, robust infrastructure services (e.g., the BPMN engine). People not yet familiar with business process modeling will first have to become acquainted with that, then BPMN4Crowd requires learning only a few additional constructs for CS; the resulting complexity is only slightly larger than that of pure BPMN.

Of course, the choice of BPMN as a starting point for the modeling of CS processes also comes with a cost: the need to stick to the abstractions and conventions of the language. This implies that not all development aspects are represented graphically and instead may require the joint use of modeling constructs and configuration parameters. This is, for instance, the case of our crowd tasks that come with a set of parameters to configure the URL of the task page, the validation process to be used, and so forth. Considering that this is common practice in BPMN, we are confident that the benefits and abstractions of the language outweigh this apparent limitation.

With CC, we propose a new type of CS platform that comes more in the form of a library of basic CS APIs and less as a self-sufficient platform. Only the implementation of suitable CS tactics brings CC to life. However, the current shortcoming of CC is that it does not yet have its own crowd-of course, this is because it is a research prototype more than a commercial tool. This limits the possibility to test the execution of CS processes with real workers. To overcome this problem and enable the crowdsourcer to be immediately productive, we started integrating CC with existing platforms, such as Amazon Mechanical Turk, and enable posting tasks on external platforms. The prototype implementation shown in Figure 19, for instance, features a TurkTask task type. Although this provides access to an existing crowd, it also introduces a limitation: it is not possible to implement one's own tactics for this kind of task, as they are hard coded inside the target platforms. 
Regarding the implementation of crowd tasks, our choice is to ask the crowdsourcer to create his own UIs for tasks via separate Web pages that can be included in the $\mathrm{CC}$ front-end. On the one hand, this gives the crowdsourcer the freedom to create and fine-tune UIs that best suit the tasks to be crowdsourced (this is a crucial aspect for the success with CS) and full control over the published and collected data. On the other hand, this requires some programming effort to create the task pages and set up the exchange of metadata between the pages and CC. This may hinder the adoption of CC by less-skilled crowdsourcers. To support this kind of crowdsourcer, we aim to develop a dedicated, hosted tool that helps crowdsourcers create task UIs and provides a set of ready-to-use page templates that can easily be configured and reused inside CS processes.

Finally, the focus of this work specifically has been on CS processes-that is, on the control flow perspective of advanced CS scenarios and less on declarative aspects such as advanced worker selection policies. The current implementation provides for worker selection based on workers' profile properties. However, it is not yet able to express more complex constraints, such as the four eyes principle ("the performer of one task cannot participate in a validation task of its own work"). Enforcing this kind of constraint requires maintaining not only worker profile information but also the history of the task instances and the specific data items on which they have worked. The metadata model implemented by CC already accommodates this kind of tracking, and the necessary API support still needs to be implemented.

\section{RELATED WORK}

The work presented in this article proposes a language and a platform to create and enact CS processes that are composed of crowd, data, machine, and human tasks. In the literature, similar solutions have been proposed, interpreting the same problem along three different lines of thought: procedural programming, parallel computing, and process modeling.

\subsection{Procedural Programming Approaches}

The procedural programming approaches mostly extend existing programming languages or define new languages able to cover CS aspects. For instance, TurKit [Little et al. 2010a] is a programming language based on JavaScript that adds support for human computation. TurKit uses Amazon Mechanical Turk as a platform to execute human tasks. Programmers can write software applications that use both human and machine computations. AutoMan [Barowy et al. 2012] is another system for human computation that integrates crowd tasks into Scala.

These works abstract CS logic at a programming language level. Programming languages allow crowdsourcers to create flexible CS logics, yet they neglect support for tactic definitions and configurations. As we showed in our case study, implementing a CS process generally is not an easy task, even with help from dedicated frameworks. Crowdsourcers who rely on programming languages are forced to code all aspects of their CS process-a task that is not trivial and makes it harder to maintain and evolve processes. In addition, the use of procedural programming is limited to programmers and is out of the reach of those without the necessary software development skills.

\subsection{Parallel Computing Approaches}

The parallel computing approaches interpret a crowd task as a complex process that can be decomposed into a set of smaller and simpler tasks that can be executed in parallel. CrowdForge [Kittur et al. 2011] and Turkomatic [Kulkarni et al. 2012] adapt, for example, the MapReduce approach [Dean and Ghemawat 2008] to crowdsource complex work. Both frameworks model tasks as a set of split and recombine tasks 
executed by workers. Workers have the possibility to solve a task or split it in smaller tasks (subtasks), in which case the workers are in charge of recombining the solutions of the subtasks into a unique solution. Jabberwocky [Ahmad et al. 2011] implements a similar MapReduce approach. Jabberwocky is composed of three different pieces: Dormouse provides operations to interact with machines and humans; ManReduce, similar to CrowdForge and Turkomatic, implements the MapReduce algorithm using Dormouse as workers; and Dog, a scripting language that can be used to specify the details of applications (e.g., defining users or task goals).

The works of this type give one the possibility to bring human and machine computations together into a single application. Compared to our approach, these systems focus on CS processes composed of parallel executions of tasks, which can be implemented as well with BPMN4Crowd, but they neglect support for more complex, generic process logics. Most of the discussed approaches require some level of scripting expertise, which is an abstraction that may not be suitable for crowdsourcers who are business analysts or those who are not programming experts.

\subsection{Process Modeling Approaches}

The process modeling approaches are most similar to the interpretation that we follow in this article. CrowdWeaver [Kittur et al. 2012], for instance, is a process modeling tool built on top of CrowdFlower. The CrowdWeaver system offers a visual tool with a graphical modeling notation to create and execute data-driven processes bringing together machine and crowd tasks, the former providing data transformation capabilities. The system abstracts the native operations and logic of CrowdFlower; it does not support crowd tactics, other CS platforms, or the integration with legacy business processes. CrowdLang [Minder and Bernstein 2011, 2012] is a model-driven language for the modeling of generic human computations. Similarly to BPMN4Crowd, it allows one to express data and control flows and describe application logics as tasks executed by the crowd or machines. CrowdLang also supports operations for the management of data. Its modeling notation features workflow modeling constructs such as rounded rectangles for tasks and diamonds for conditions, but it also introduces additional constructs, such as the decision, which is modeled with a circle shape. More importantly, each task instance in CrowdLang must be modeled as an individual task, and thus crowdsourcers must create a task in the model for each task instance they need. This may result in a huge number of similar tasks and is not very efficient from a modeling perspective. Bozzon et al. [2014] propose methods and tools for designing crowd-based workflows as interacting tasks and additionally propose a set of typical workflow patterns that help crowdsourcers implement their CS processes (BPMN4Crowd is also powerful in expressing reusable logics and patterns). The proposed modeling language is accompanied by a runtime environment called CrowdSearcher that supports deploying tasks on both CS platforms and social networks and monitoring CS processes at runtime. The environment also provides explicit support for data storage and processing.

The three approaches are suitable for modeling CS processes. However, they do not provide crowdsourcers with the flexibility to also define their own tactics. In addition, they all feature a proprietary modeling notation that may not be straightforward to crowdsourcers. We instead extend BPMN and support the integration with most of the state-of-the-art BPM tools, which makes modeling more accessible to people who already know BPMN and integration into legacy processes easier.

Many researchers [Curran et al. 2009; Vukovic 2009; La Vecchia and Cisternino 2010; Kittur et al. 2013] have highlighted the benefits of the integration of systems that manage human- and machine-based work with BPM or workflow management in CS. This integration can be achieved in various ways, next to the one that we describe in this article. For example, Khazankin et al. [2012] present an approach 
to an adaptable execution of business processes on top of a CS platform. Its main peculiarity is the ability to determine optimal task publishing procedures, adapting them to modeled process deadlines and minimizing the reward cost. The approach optimizes process execution, which is a feature that CC could integrate in the future and from which it could benefit. Skopik et al. [2011] use Human-Provided Services (HPS) [Schall et al. 2008], which abstract human capabilities as Web services, easing the interaction with people and the integration with SOA systems. The supported processes use crowd tasks that are executed by people taken from a social network. Schall et al. [2012] extend BPEL4People [Active Endpoints, Adobe, BEA, IBM, Oracle, SAP 2007], an extension of the Web service orchestration language BPEL [Jordan and Evdemon 2007], with parameters to specify requirements specific to CS, such as user skills and deadlines. Both approaches tackle the problem of CS by abstracting worker capabilities as configurations of tasks or services.

In a more general context, human computation - that is, the use of human capabilities to solve problems - recently has been investigated and adopted as a way to solve tasks that machines cannot solve. CS as proposed by Howe [2008] is one possible example of human computation. Another example of human computation is social computing, which studies and leverages on social human behaviors facilitated by computers (examples are blogs and wikis) [Quinn and Bederson 2011]. This line of thought has inspired a practice called social BPM, which is a recent trend in research that fuses social interactions as enabled by social software and BPM [Johannesson et al. 2009; Erol et al. 2010]. BPM is a multifaceted domain, and social capabilities have been used, for instance, to improve the design of processes [Koschmider et al. 2010] or enable the coordination and collaboration of multiple actors during process execution [Dengler et al. 2011]. Then there are social BPM approaches that extend business process languages, similar to our approach, but leverage on the capabilities of people acting in generic social networks rather than in dedicated CS platforms. BPM4People [Brambilla et al. 2011, 2012] proposes, for instance, a set of extensions of BPMN that enable the modeling and deployment of process-aware, social interactions over social networks, such as the collection of votes or comments. BPM4People supports a social computing paradigm in which work is mostly executed implicitly, and actors may not be aware that they are taking part in a task or work. Instead, our focus is on CS platforms, where tasks are defined explicitly and possibly rewarded and where actors participate consciously either to offer or to execute work.

\section{CONCLUSION}

This article advances the state of the art on CS along three core directions. First, it establishes $C S$ processes as processes that involve individual actors, machines, and workers as first-class development concern, going beyond the conventional focus on crowd tasks only. Second, it proposes an alternative interpretation of the CS platformCC_-that comes as a set of CS-specific APIs and allows the crowdsourcer to implement custom CS tactics for the CS of individual tasks. Third, it fosters the integration of CS capabilities with state-of-the-art BPM practices and legacy systems. CS processes allow the crowdsourcer to specify advanced CS and process logics at a level of abstraction that has proven to be suitable for the specification and coordination of tasks (i.e., business processes). CS tactics provide the crowdsourcer with unprecedented control over how work is advertised, assigned, executed, integrated, evaluated, and rewarded. The use of off-the-shelf BPM technology finally makes the two CS instruments available as a service that can be integrated flexibly into existing software.

The tangible contributions of the article are (1) a prototype implementation of $\mathrm{CC}$ available as open-source software, (2) an extension of BPMN-BPMN4Crowdspecifically tailored to the needs of CS, (3) a set of reusable tactic process models, 
(4) a set of reusable validation process models, (5) a visual editor for the modeling of BPMN4Crowd processes, and (6) a case study that discusses the benefits and shortcomings of the approach as a whole in the context of a concrete human computation scenario.

In future work, we plan to provide an online repository where crowdsourcers can search for, retrieve, and share tactics, validation processes, and CS processes to foster knowledge reuse and exchange. Similarly, we are considering the implementation of a hosted instrument for the visual design of crowd task pages, starting from a set of predefined templates for recurrent task types, such as questionnaires, comparisons, voting tasks, and text translations. We also intend to use CC and BPMN4Crowd ourselves for the design of other CS experiments, such as the case study described in this article.

\section{ACKNOWLEDGMENTS}

We are grateful to Carlos Rodríguez (University of Trento) for the fruitful feedback and for providing insight into the case study on mining mashup model patterns from Yahoo! Pipes.

\section{REFERENCES}

Active Endpoints, Adobe, BEA, IBM, Oracle, SAP. 2007. WS-BPEL Extension for People (BPEL4People) Version 1.0. Technical Report.

Salman Ahmad, Alexis Battle, Zahan Malkani, and Sepander Kamvar. 2011. The Jabberwocky programming environment for structured social computing. In Proceedings of the 24th Annual ACM Symposium on User Interface Software and Technology (UIST'11). 53-64.

Mohammad Allahbakhsh, Boualem Benatallah, Aleksandar Ignjatovic, Hamid Reza Motahari-Nezhad, Elisa Bertino, and Schahram Dustdar. 2013. Quality control in crowdsourcing systems: Issues and directions. IEEE Internet Computing 17, 2, 76-81.

Daniel W. Barowy, Charlie Curtsinger, Emery D. Berger, and Andrew McGregor. 2012. AutoMan: A platform for integrating human-based and digital computation. ACM SIGPLAN Notices 47, 10, 639-654.

Christian Baun, Marcel Kunze, Jens Nimis, and Stefan Tai. 2011. Cloud Computing: Web-Based Dynamic IT Services. Springer.

Alessandro Bozzon, Marco Brambilla, Stefano Ceri, Andrea Mauri, and Riccardo Volonterio. 2014. Patternbased specification of crowdsourcing applications. In Web Engineering. Lecture Notes in Computer Science, Vol. 8541. Springer, 218-235.

Marco Brambilla, Piero Fraternali, and Carmen Vaca. 2011. A notation for supporting social business process modeling. In Business Process Model and Notation. Lecture Notes in Business Information Processing, Vol. 95. Springer, 88-102.

Marco Brambilla, Piero Fraternali, and Carmen Karina Vaca Ruiz. 2012. Combining social Web and BPM for improving enterprise performances: The BPM4People approach to social BPM. In Proceedings of the 21st International Conference Companion on World Wide Web (WWW'12 Companion). 223-226.

Ruggiero Cavallo and Shaili Jain. 2012. Efficient crowdsourcing contests. In Proceedings of the 11th International Conference on Autonomous Agents and Multiagent Systems-Volume 2 (AAMAS'12). 677-686.

Crowdsourcing Week. 2014. The 2014 Global Crowdsourcing Pulsecheck: 1st Annual Survey Topline Results. Retrieved April 19, 2015, from http://www.slideshare.net/crowdsourcingweek/2014-globalcrowdsourcing-pulsecheck-1st-annual-survey-topline-results.

Stephan Curran, Kevin Feeney, Reinhard Schaler, and David Lewis. 2009. The management of crowdsourcing in business processes. In Proceedings of the IFIP/IEEE International Symposium on Integrated Network Management-Workshops, 2009. 77-78.

Florian Daniel and Maristella Matera. 2014. Mashups: Concepts, Models and Architectures. Springer.

Jeffrey Dean and Sanjay Ghemawat. 2008. MapReduce: Simplified data processing on large clusters. Communications of the ACM 51, 1, 107-113.

Frank Dengler, Agnes Koschmider, Andreas Oberweis, and Huayu Zhang. 2011. Social software for coordination of collaborative process activities. In Proceedings of the 3rd Workshop on Business Process Management and Social Software. 396-407. 
Steven Dow, Anand Kulkarni, Scott Klemmer, and Björn Hartmann. 2012. Shepherding the crowd yields better work. In Proceedings of the ACM 2012 Conference on Computer Supported Cooperative Work (CSCW'12). 1013-1022.

Selim Erol, Michael Granitzer, Simone Happ, Sami Jantunen, Ben Jennings, Paul Johannesson, Agnes Koschmider, Selmin Nurcan, Davide Rossi, and Rainer Schmidt. 2010. Combining BPM and social software: Contradiction or chance? Journal of Software Maintenance and Evolution: Research and Practice $22,67,449-476$.

Michael J. Franklin, Donald Kossmann, Tim Kraska, Sukriti Ramesh, and Reynold Xin. 2011. CrowdDB: Answering queries with crowdsourcing. In Proceedings of the 2011 ACM SIGMOD International Conference on Management of Data. 61-72.

Matthias Hirth, Tobias Hoßfeld, and Phuoc Tran-Gia. 2013. Analyzing costs and accuracy of validation mechanisms for crowdsourcing platforms. Mathematical and Computer Modelling 57, 11-12, 29182932.

Tobias Hoßfeld, Matthias Hirth, Pavel Korshunov, Philippe Hanhart, Bruno Gardlo, Christian Keimel, and Christian Timmerer. 2014a. Survey of Web-based crowdsourcing frameworks for subjective quality assessment. In Proceedings of the 2014 IEEE 16th International Workshop on Multimedia Signal Processing (MMSP'14). 1-6.

Tobias Hoßfeld, Matthias Hirth, and Phuoc Tran-Gia. 2011. Modeling of crowdsourcing platforms and granularity of work organization in Future Internet. In Proceedings of the 201123 rd International Teletraffic Congress (ITC'11). 142-149.

Tobias Hoßfeld, Christian Keimel, Matthias Hirth, Bruno Gardlo, Julian Habigt, Klaus Diepold, and Phuoc Tran-Gia. 2014b. Best practices for QoE crowdtesting: QoE assessment with crowdsourcing. IEEE Transactions on Multimedia 16, 2, 541-558.

Jeff Howe. 2008. Crowdsourcing: Why the Power of the Crowd Is Driving the Future of Business. Crown Publishing Group, New York, NY.

Panagiotis G. Ipeirotis. 2010. Analyzing the Amazon Mechanical Turk marketplace. XRDS: Crossroads, the ACM Magazine for Students 17, 2, 16-21.

Panagiotis G. Ipeirotis, Foster Provost, and Jing Wang. 2010. Quality management on Amazon Mechanical Turk. In Proceedings of the ACM SIGKDD Workshop on Human Computation (HCOMP'10). ACM, New York, NY, 64-67.

Paul Johannesson, Birger Andersson, and Petia Wohed. 2009. Business process management with social software systems-a new paradigm for work organisation. In Business Process Management Workshops. Lecture Notes in Business Information Processing. Springer, 659-665.

Diane Jordan and John Evdemon. 2007. Web Services Business Process Execution Language Version 2.0. OASIS. http://docs.oasis-open.org/wsbpel/2.0/OS/wsbpel-v2.0-OS.html.

Roman Khazankin, Benjamin Satzger, and Schahram Dustdar. 2012. Optimized execution of business processes on crowdsourcing platforms. In Proceedings of the 2012 8th International Conference on Collaborative Computing: Networking, Applications, and Worksharing (CollaborateCom'12). 443-451.

Aniket Kittur, Susheel Khamkar, Paul André, and Robert Kraut. 2012. Crowdweaver: Visually managing complex crowd work. In Proceedings of the ACM 2012 Conference on Computer Supported Cooperative Work (CSCW'12). ACM, New York, NY, 1033-1036.

Aniket Kittur, Jeffrey V. Nickerson, Michael Bernstein, Elizabeth Gerber, Aaron Shaw, John Zimmerman, Matt Lease, and John Horton. 2013. The future of crowd work. In Proceedings of the 2013 Conference on Computer Supported Cooperative Work (CSCW'13). ACM, New York, NY, 1301-1318.

Aniket Kittur, Boris Smus, Susheel Khamkar, and Robert E. Kraut. 2011. CrowdForge: Crowdsourcing complex work. In Proceedings of the 24th Annual ACM Symposium on User Interface Software and Technology (UIST'11). 43-52.

Agnes Koschmider, Minseok Song, and Hajo A. Reijers. 2010. Social software for business process modeling. Journal of Information Technology 25, 3, 308-322.

Pavel Kucherbaev, Stefano Tranquillini, Florian Daniel, Fabio Casati, Maurizio Marchese, Marco Brambilla, and Piero Fraternali. 2013. Business processes for the Crowd Computer. In Business Process Management Workshops. Lecture Notes in Business Information Processing, Vol. 132. Springer, 256267.

Anand Kulkarni, Matthew Can, and Björn Hartmann. 2012. Collaboratively crowdsourcing workflows with turkomatic. In Proceedings of the ACM 2012 Conference on Computer Supported Cooperative Work (CSCW'12). ACM, New York, NY, 1003-1012.

Gioacchino La Vecchia and Antonio Cisternino. 2010. Collaborative workforce, business process crowdsourcing as an alternative of BPO. In Proceedings of the 10th International Conference on Current Trends in Web Engineering (ICWE'10). 425-430. 
Greg Little, Lydia B. Chilton, Max Goldman, and Robert C. Miller. 2010a. Exploring iterative and parallel human computation processes. In Proceedings of the ACM SIGKDD Workshop on Human Computation (HCOMP'10). ACM, New York, NY, 68-76.

Greg Little, Lydia B. Chilton, Max Goldman, and Robert C. Miller. 2010b. TurKit: Human computation algorithms on Mechanical Turk. In Proceedings of the 23rd Annual ACM Symposium on User Interface Software and Technology (UIST'10). ACM, New York, NY, 57-66.

Massolution. 2013. The Crowd in the Cloud: Exploring the Future of Outsourcing. White Paper. Massolution.

Patrick Minder and Abraham Bernstein. 2011. CrowdLang-first steps towards programmable human computers for general computation. In Proceedings of the Workshops at the 25th AAAI Conference on Artificial Intelligence.

Patrick Minder and Abraham Bernstein. 2012. CrowdLang: A programming language for the systematic exploration of human computation systems. In Social Informatics. Lecture Notes in Computer Science, Vol. 7710. Springer, 124-137.

David Oleson, Alexander Sorokin, Greg P. Laughlin, Vaughn Hester, John Le, and Lukas Biewald. 2011. Programmatic gold: Targeted and scalable quality assurance in crowdsourcing. In Proceedings of the Workshops at the 25th AAAI Conference on Artificial Intelligence.

Object Management Group. 2011. Business Process Model and Notation (BPMN) Version 2.0. Available at http://www.omg.org.

Alexander J. Quinn and Benjamin B. Bederson. 2011. Human computation: A survey and taxonomy of a growing field. In Proceedings of the SIGCHI Conference on Human Factors in Computing Systems (CHI'11). ACM, New York, NY, 1403-1412.

Carlos Rodriguez, Florian Daniel, and Fabio Casati. 2014. Crowd-based mining of reusable process model patterns. In Business Process Management. Lecture Notes in Computer Science, Vol. 8659. Springer, 51-66.

Carlos Rodriguez, Eros Zaupa, Florian Daniel, and Fabio Casati. 2013. Crowd-Based Pattern Mining: On the Crowdsourcing of Reusable Knowledge Identification from Mashup Models. UNITN.

Soudip Roy Chowdhury, Florian Daniel, and Fabio Casati. 2011. Efficient, interactive recommendation of mashup composition knowledge. In Proceedings of the 9th International Conference on Service Oriented Computing (ICSOC'11). 374-388.

Soudip Roy Chowdhury, Carlos Rodríguez, Florian Daniel, and Fabio Casati. 2012. Baya: Assisted mashup development as a service. In Proceedings of the 21st International Conference Companion on World Wide Web (WWW'12). ACM, New York, NY, 409-412.

Benjamin Satzger, Harald Psaier, Daniel Schall, and Schahram Dustdar. 2013. Auction-based crowdsourcing supporting skill management. Information Systems 38, 4, 547-560.

Daniel Schall, Benjamin Satzger, and Harald Psaier. 2012. Crowdsourcing tasks to social networks in BPEL4People. World Wide Web 17, 1, 1-32.

Daniel Schall, Hong-Linh Truong, and Schahram Dustdar. 2008. Unifying human and software services in web-scale collaborations. IEEE Internet Computing 12, 3, 62-68.

Florian Skopik, Daniel Schall, Harald Psaier, Martin Treiber, and Schahram Dustdar. 2011. Towards social crowd environments using service-oriented architectures. Information Technology 53, 3, 108-116.

Mohammad Soleymani and Martha Larson. 2010. Crowdsourcing for affective annotation of video: Development of a viewer-reported boredom corpus. In Proceedings of the ACM SIGIR 2010 Workshop on Crowdsourcing for Search Evaluation (CSE'10). 4-8.

Maja Vukovic. 2009. Crowdsourcing for enterprises. In Proceedings of the 2009 World Conference on Services. $686-692$.

Mathias Weske. 2007. Business Process Management: Concepts, Languages, Architectures. Springer.

Received July 2014; revised December 2014; accepted March 2015 